\title{
Determination of the Stress State of a Piecewise Homogeneous Elastic Body with a Row of Cracks on an Interface Surface Subject to Antiplane Strains with Inclusions at the Tips
}

\author{
Ali Golsoorat Pahlaviani and Suren Mkhitaryan \\ Institute of Mechanics, National Academy of Sciences, 24 b M. Baghramian Avenue, 0019 Yerevan, Armenia \\ Correspondence should be addressed to Ali Golsoorat Pahlaviani; rahsazehpol@yahoo.com
}

Received 6 January 2015; Accepted 11 March 2015

Academic Editor: Christopher Pretty

Copyright (C) 2015 A. Golsoorat Pahlaviani and S. Mkhitaryan. This is an open access article distributed under the Creative Commons Attribution License, which permits unrestricted use, distribution, and reproduction in any medium, provided the original work is properly cited.

\begin{abstract}
The stress state of a bimaterial elastic body that has a row of cracks on an interface surface is considered. It is subjected to antiplane deformations by uniformly distributed shear forces acting on the horizontal sides of the body. The governing equations of the problem, the stress intensity factors, the deformation of the crack edges, and the shear stresses are derived. The solution of the problem via the Fourier sine series is reduced to the determination of a singular integral equation (SIE) and consequently to a system of linear equations. In the end, the problem is solved in special cases with inclusions. The results of this paper and the previously published results show that the used approach based on the Gauss-Chebyshev quadrature method can be considered as a generalized procedure to solve the collinear crack problems in mode I, II, or III loadings.
\end{abstract}

\section{Introduction}

Stress analysis near a fracture in an elastic material is one of the most explored topics in solid mechanics. Calculation and stress analysis of engineering structures, particularly their connections and the determination of the stress and strain distribution fields of cracked bodies, have received attention from numerous investigators in recent years. The stress intensity factors, shear stresses, and crack opening displacements are major concepts that must be determined. The stress intensity factor is an important parameter that denotes the magnitude of the stress singularity. The singular order is a single real value, for example, 0.5 for a crack in a homogeneous material. The singular order of general interface corners may be real or complex. An asymptotic stress near the tip of a sharp interfacial corner is generally singular as a result of a mismatch between the materials' elastic constants.

In this paper, the problem of a piecewise homogeneous rectangular prismatic elastic body in an antiplane strain state due to antiplane forces is discussed. The plate is composed of two bonded dissimilar materials with a number of arbitrary collinear cracks on their interface surface. The aim of this work is the derivation of analytical expressions for the stress intensity factors of the cracks and the presentation of a new mathematical-numerical approach to solve singular integral equations related to the beginning of stresses at the tips of cracks in antiplane deformations; thus, a numerical method to calculate the SIFs of an interface crack between dissimilar materials is developed. Calculation of the SIFs for bimaterial plates in dimensionless form is conducted for several cases: a row of cracks, one and two cracks, and one crack with inclusions at the tips. The objectives of the present study are (i) to present a new method for calculating SIFs of interfacial cracks subject to antiplane loading and (ii) to investigate the influence of inclusion moduli on SIFs to reduce SIFs in cracked bodies and to direct us towards a method for repairs.

The existence of three-dimensional effects at cracks has been known for many years, but understanding has been limited, and for some situations, it still is. Understanding improved when the existence of corner point singularities and their implications became known for straight through-thethickness cracks $[1,2]$.

It has been known for a long time that shear and antiplane fracture modes are coupled. This means that shear 
or antiplane loading of an elastic plate with a throughthe-thickness crack generates a coupled three-dimensional antiplane or shear singular stress state, respectively. These singular stress states (or coupled fracture modes) are currently largely ignored in theoretical and experimental investigations as well as in standards and failure assessment codes of structural components, in which it is implicitly assumed that the intensities of these modes as well as other three-dimensional effects are negligible in comparison with the stress fields generated by the primary modes (modes I, II, and III) [3, 4].

The theoretical bases of fracture stresses are discussed in the literature [5-7], and the conclusions of numerous studies and investigations on the derivation of SIFs are categorized in $[8-10]$. Most of these studies were performed on homogeneous plates. It is proven that, under certain circumstances, the three-dimensional governing equations of elasticity can be reduced to a system where a biharmonic equation and a harmonic equation have to be simultaneously satisfied. The former provides the solution of the corresponding plane problem, while the latter provides the solution of the corresponding out-of-plane shear problem [11]. On the other hand, a mixed fracture mode under antiplane loading may also occur. This coupled fracture mode represents one of threedimensional phenomena that are currently largely ignored in numerical simulations and failure assessments of structural components weakened by cracks. It arises due to the boundary conditions on the plate-free surfaces, which negate the transverse shear stress components corresponding to classical mode III. Instead, a new singular stress state in addition to the well-known 3D corner singularity is generated. This singular stress state can affect or contribute significantly to the fracture initiation conditions $[12,13]$.

Inclusions and cavities are also important in understanding the mechanical behavior of structures and are studied in several papers, for example, 2D linear elastic materials [14] and antiplane shear cracks [15]. Photoelasticity and finite element methods have also been employed to study the interaction between collinear cracks, and good agreement was found [16]. Photoelasticity is very helpful in investigating the stress state near inclusions. The results show that the singular stress field predicted by the linear elastic solution for an inclusion can be generated in reality with great accuracy [17]. The inclusions form a thin material that constituted a rigid line inclusion, embedded in a linear elastic body to produce an inhomogeneous stress state. The experiments fully validate the stress state calculated for an elastic plate [18].

The mechanical behavior of thin inclusions is fundamental to the design of composite materials. It is realized that, for a given geometry and boundary condition, $K_{\mathrm{III}}$ depends on the gradation of both the modulus of elasticity and Poisson's ratio [19]. The cracked sandwich plate twist specimen is viable to characterize mode III fracture [20].

The stress intensity factors can be calculated by a pathindependent h-integral and through the virtual crack closureintegral method (VCCM) for numerical implementation [21]. The present study is aimed at investigating the stress state of a piecewise homogeneous elastic body which has a row of collinear cracks in mode III. The numerical procedure based on the loading Gauss-Chebyshev quadrature method

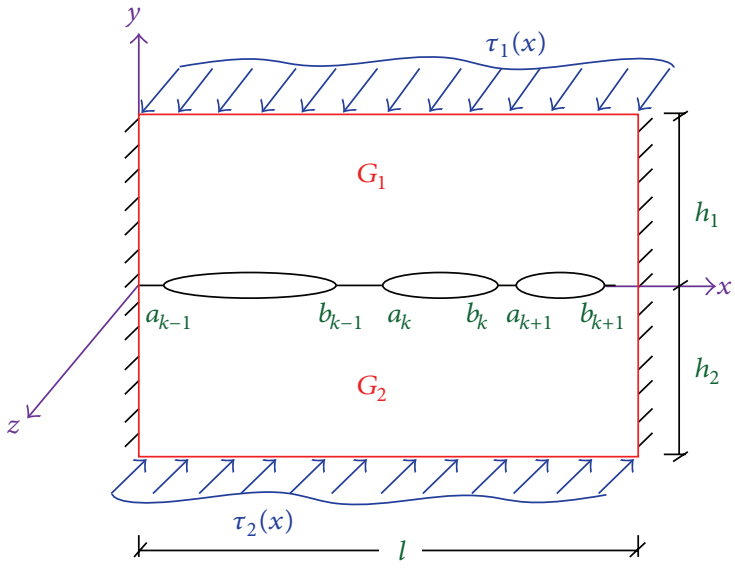

FIGURE 1: Rectangular piecewise homogeneous elastic body with several collinear cracks.

is applied. This approach can be used for other multicrack problems or more complicated types of loading $[22,23]$.

At the end of this paper, we discuss the influence of the bimaterial nature of the body, the distance and geometry of the cracks, and the presence of inclusions at the tips on the characteristics of antiplane shear stresses and deformations.

\section{Derivation of the Singular Integral Equation for the General Form of the Problem}

A piecewise homogeneous rectangular body in the Cartesian coordinate system $O x y z$ is considered as shown in Figure 1.

In the equations below, subscript 1 denotes rectangular plate $D_{1}$ and subscript 2 denotes $D_{2}$.

The top rectangular plate $D_{1}=\left\{0 \leq x \leq l ; 0 \leq y \leq h_{1}\right\}$ has a rigidity modulus $G_{1}$, length $l$, and height $h_{1}$, and the bottom rectangular plate $D_{2}=\left\{0 \leq x \leq l ;-h_{2} \leq y \leq 0\right\}$ has a rigidity modulus $G_{2}$, length $l$, and height $h_{2}$. On the bonding surface of the two materials in the interval $0 \leq x \leq l$, there are $N$ arbitrary collinear cracks with total length $L$ and individual lengths $L_{k}$; that is,

$$
\begin{gathered}
L_{k}=\left[a_{k}, b_{k}\right] \quad(k=\overline{1, N})\left(a_{1} \geq 0, b_{N} \leq l\right), \\
L=\bigcup_{k=1}^{N} L_{k}=\bigcup_{k=1}^{N}\left[a_{k}, b_{k}\right], \\
\left(a_{k}<b_{k}(k=\overline{1, N}), b_{k}<a_{k+1}(k=\overline{1, N-1})\right) .
\end{gathered}
$$

The upper and lower boundaries of these cracks are subjected to antiplane shear stresses $\tau_{ \pm}^{(0)}(x)$, which act as varying internal loadings of openings:

$$
\begin{gathered}
\left.\tau_{y z}^{(1)}\right|_{y=+0}=\tau_{+}^{(0)}(x) \quad\left(x \in L^{+}\right) ; \\
\left.\tau_{y z}^{(2)}\right|_{y=-0}=\tau_{-}^{(0)}(x) \quad\left(x \in L^{-}\right), \\
L^{ \pm}=\bigcup_{k=1}^{N} L_{k}^{ \pm} .
\end{gathered}
$$


The edges $x=0$ and $x=l$ of rectangles $D_{j}(j=1,2)$ are restricted, and the edges of the rectangles $y=h_{1}$ and $y=-h_{2}$ are subjected to antiplane shear stresses: $\tau_{j}(x)(j=1,2)$,

$$
\begin{array}{r}
\left.\tau_{y z}^{(1)}\right|_{y=h_{1}-0}=\tau_{1}(x),\left.\quad \tau_{y z}^{(2)}\right|_{y=-h_{2}+0} \\
=\tau_{2}(x) \\
(0<x<l),
\end{array}
$$

where the notation $\tau_{y z}^{(j)}(j=1,2)$ for shearing stress components in plates $D_{j}$ is used. Because the pair of vertical sides of the rectangle is rigidly fixed, the boundary value problem for the complex potential of state can be transformed to the problem in the half-plane, and its solution can be obtained in quadrature using conformal mapping [24, 25].

Through the above assumptions, the body is under the antiplane strain condition, so the displacements of the crack edges are along the $O z$-axis with the base on the $O x y$ surface.

It is required to calculate the dislocation density of the crack boundaries, the related stress intensity factors, and the distribution of shearing stresses in the regions that are outside of the crack system, with total length $L^{\prime}=[0, l] \backslash L$.

For the development of the above-mentioned functions, the plate is divided into an upper rectangle $\left(D_{1}\right)$ and a lower rectangle $\left(D_{2}\right)$ on the $O x$-axis with $0 \leq x \leq l$, and we introduce a function $w_{j}(x, y)(j=1,2)$ as the only nonzero displacement component along the $O z$-axis for both $D_{j}$ $(j=1,2)$ rectangular plates and separately investigate their individual elastic equilibria. In this manner, for rectangle $\left(D_{1}\right)$, according to Hooke's law, the below boundary value problem is found:

$$
\begin{gathered}
\Delta w_{1}=\frac{\partial^{2} w_{1}}{\partial x^{2}}+\frac{\partial^{2} w_{1}}{\partial y^{2}}=0 \quad\left(0<x<l, 0<y<h_{1}\right), \\
\left.w_{1}(x, y)\right|_{x=0}=\left.w_{1}(x, y)\right|_{x=l}=0 \quad\left(0<y<h_{1}\right), \\
\left.\tau_{y z}^{(1)}\right|_{y=+0}=\left.G_{1} \frac{\partial w_{1}}{\partial y}\right|_{y=+0}=\tau_{+}(x), \\
\left.\tau_{y z}^{(1)}\right|_{y=h_{1}}=\left.G_{1} \frac{\partial w_{1}}{\partial y}\right|_{y=h_{1}}=\tau_{1}(x) \quad(0<x<l) .
\end{gathered}
$$

Suppose the notation

$$
\left.\tau_{y z}^{(1)}\right|_{y=+0}=\tau_{+}(x)= \begin{cases}\tau_{+}^{(0)}(x) & \left(x \in L^{+}\right) \\ \tau(x) & \left(x \in L^{\prime}\right)\end{cases}
$$

in which the function $\tau_{+}^{(0)}(x)$, as mentioned before, is the shear stress loading on the cracks' top boundaries $L^{+}$and $\tau(x)$ is the unknown tearing shear stress that acts on the noncracked interface surface system $L^{\prime}$.

According to [26], to solve (4) by means of the Fourier sine series, it is obtained that

$$
\bar{w}_{1}(n, y)=\int_{0}^{l} w_{1}(x, y) \sin \left(\frac{\pi n x}{l}\right) d x \quad(n=1,2, \ldots) .
$$

Hence from the inverse Fourier series we deduce

$$
w_{1}(x, y)=\frac{2}{l} \sum_{n=1}^{\infty} \bar{w}_{1}(n, y) \sin \left(\frac{\pi n x}{l}\right) \quad(0 \leq x \leq l) .
$$

Equation (7) is the Fourier sine series with coefficients given by (6). The reason for using the Fourier sine series is that boundary value problem (4) with the Laplacian and Dirichlet boundary conditions can be solved by separation of variables, and because the boundary conditions in the $x$ variable are the Dirichlet boundary conditions, this generates a Fourier sine series with eigenfunctions $\sin (n \pi x / l)$ and eigenvalues $(n \pi x / l)^{2}, n=1,2, \ldots$

Considering (6) and (7), it follows that by taking the two parts of the differential equation (4), multiplying by $\sin (\pi n x / l)$, integrating it over the interval $(0, l)$, and taking into consideration the boundary condition (4), we obtain the below boundary value problem:

$$
\begin{gathered}
\frac{d^{2} \bar{w}_{1}}{d x^{2}}-\frac{\pi^{2} n^{2}}{l^{2}} \bar{w}_{1}=0 \quad\left(0<y<h_{1}\right), \\
\left.G_{1} \frac{d \bar{w}_{1}}{d y}\right|_{y=+0}=\bar{\tau}_{+}(n),\left.\quad G_{1} \frac{d \bar{w}_{1}}{d y}\right|_{y=h_{1}}=\bar{\tau}_{1}(n)
\end{gathered}
$$

with

$$
\begin{aligned}
& \bar{\tau}_{+}(n)=\int_{0}^{l} \tau_{+}(x) \sin \left(\frac{\pi n x}{l}\right) d x, \\
& \bar{\tau}_{1}(n)=\int_{0}^{l} \tau_{1}(x) \sin \left(\frac{\pi n x}{l}\right) d x .
\end{aligned}
$$

Boundary value problem (8) can be solved by the following formula:

$$
\begin{aligned}
\bar{w}_{1}(n, y)= & \frac{l}{\pi n G_{1} \sin \left(\pi n h_{1} / l\right)} \\
& \times\left\{\bar{\tau}_{1}(n) \operatorname{ch}\left(\frac{\pi n y}{l}\right)\right. \\
& \left.-\bar{\tau}_{+}(n) \operatorname{ch}\left(\frac{\pi n\left(y-h_{1}\right)}{l}\right)\right\} \\
& \left(0 \leq y \leq h_{1}\right) .
\end{aligned}
$$

For $(n=1,2, \ldots)$, we can conclude

$$
\bar{w}_{1}(n, 0)=\frac{l\left[\bar{\tau}_{1}(n)-\bar{\tau}_{+}(n) \operatorname{ch}\left(\pi n h_{1} / l\right)\right]}{\pi n G_{1} \operatorname{sh}\left(\pi n h_{1} / l\right)} .
$$


Using a similar approach, the boundary value problem related to the rectangular plate $\left(D_{2}\right)$ is obtained:

$$
\begin{gathered}
\bar{w}_{2}(n, 0)=\frac{l\left[\bar{\tau}_{-}(n) \operatorname{ch}\left(\pi n h_{2} / l\right)-\bar{\tau}_{2}(n)\right]}{\pi n G_{2} \operatorname{sh}\left(\pi n h_{2} / l\right)}, \\
\left\{\bar{w}_{2}(n, y), \bar{\tau}_{-}(n), \bar{\tau}_{2}(n)\right\} \\
=\int_{0}^{l}\left\{w_{2}(x, y), \tau_{-}(x), \tau_{2}(x)\right\} \\
\cdot \sin \left(\frac{\pi n x}{l}\right) d x, \\
\tau_{-}(x)=\left.\tau_{y z}^{(2)}\right|_{y=-0}= \begin{cases}\tau_{-}^{(0)}(x) & \left(x \in L^{-}\right) \\
\tau(x) & \left(x \in L^{\prime}\right) .\end{cases}
\end{gathered}
$$

Then, considering the $w_{1}(x, 0)$ and $w_{2}(x, 0)$ displacements and the following functions:

$$
\begin{aligned}
& \Phi(x)=\frac{w_{1}(x, 0)-w_{2}(x, 0)}{2}=\frac{2}{l} \sum_{n=1}^{\infty} \Phi_{n} \sin \left(\frac{\pi n x}{l}\right), \\
& \Psi(x)=\frac{w_{1}(x, 0)+w_{2}(x, 0)}{2}=\frac{2}{l} \sum_{n=1}^{\infty} \Psi_{n} \sin \left(\frac{\pi n x}{l}\right), \\
& \Omega(x)=\frac{\tau_{+}(x)+\tau_{-}(x)}{2}=\frac{2}{l} \sum_{n=1}^{\infty} \Omega_{n} \sin \left(\frac{\pi n x}{l}\right), \\
& X(x)=\frac{\tau_{+}(x)-\tau_{-}(x)}{2}=\frac{2}{l} \sum_{n=1}^{\infty} X_{n} \sin \left(\frac{\pi n x}{l}\right) \\
& \quad(0<x<l),
\end{aligned}
$$

where $\Phi_{n}, \Psi_{n}, \Omega_{n}$, and $X_{n}$ are the Fourier sine series coefficients of the above functions that are similar to those introduced in (7) and (9), we obtained

$$
\begin{aligned}
& \Phi_{n}=\frac{1}{2}\left[\bar{w}_{1}(n, 0)-\bar{w}_{2}(n, 0)\right], \\
& \Psi_{n}=\frac{1}{2}\left[\bar{w}_{1}(n, 0)+\bar{w}_{2}(n, 0)\right], \\
& \Omega_{n}=\frac{1}{2}\left[\bar{\tau}_{+}(n)+\bar{\tau}_{-}(n)\right], \\
& X_{n}=\frac{1}{2}\left[\bar{\tau}_{+}(n)-\bar{\tau}_{-}(n)\right] .
\end{aligned}
$$

Substituting coefficients (14) into (11) and (12), a system of two linear equations with respect to $\Omega_{n}$ and $\Psi_{n}$ is obtained. Solving the system, coefficients $\Omega_{n}$ and $\Psi_{n}$ can be expressed by $\Phi_{n}$ and $X_{n}$ and, in turn, all of the functions in (13) can be expressed by the $\Phi_{n}$ and $X_{n}$ coefficients. In this case, as we expect, the required function (13) after some simple transforms is found in dimensionless form as follows (see Appendix):

$$
\begin{aligned}
\Omega_{0}(\xi)= & -\frac{1}{2 \pi} \int_{L_{0}}\left(\operatorname{ctg} \frac{\xi-\eta}{2}+\operatorname{ctg} \frac{\xi+\eta}{2}\right) \varphi_{0}(\eta) d \eta \\
& +\frac{1}{\pi} \int_{L_{0}}\left[K_{*}(\xi-\eta)+K_{*}(\xi+\eta)\right] \varphi_{0}(\eta) d \eta \\
& +\frac{\mu-1}{\mu+1} \widetilde{\tau}_{+}(\xi)-\widetilde{\tau}_{-}(\xi)+\frac{2 \mu}{\pi(1+\mu)} \\
& \times \int_{L_{0}}\left[L_{*}(|\xi-\eta|)-L_{*}(\xi+\eta)\right] \\
& \left.\left.+\frac{1}{\pi} \int_{0}^{\pi}\left[\tilde{\tau}_{+}^{*}(\eta)-\widetilde{\tau}_{-}(\eta)\right] d \eta-\eta \mid\right)-L_{1}^{*}(\xi+\eta)\right] \bar{\tau}_{1}(\eta) d \eta \\
& +\frac{1}{\pi} \int_{0}^{\pi}\left[L_{2}^{*}(|\xi-\eta|)-L_{2}^{*}(\xi+\eta)\right] \bar{\tau}_{2}(\eta) d \eta,
\end{aligned}
$$

where the first part in the first integral when $\eta=\xi$ is the main quantity in the Cauchy formulation.

The other parameters are

$$
\begin{aligned}
& K_{*}(\xi)= \sum_{n=1}^{\infty} \frac{\sin (n \xi)}{\Delta_{n}\left(\mu, h_{+}, h_{-}\right)} \\
& \times\left[\mu \operatorname{sh}\left(n h_{+}\right) e^{-n h_{-}}+\operatorname{sh}\left(n h_{-}\right) e^{-n h_{+}}\right], \\
& L_{*}(\xi)= \sum_{n=1}^{\infty} \frac{\cos (n \xi)}{\Delta_{n}\left(\mu, h_{+}, h_{-}\right)} \operatorname{sh}\left[n\left(h_{+}-h_{-}\right)\right], \\
& L_{1}^{*}(\xi)=\sum_{n=1}^{\infty} \frac{\cos (n \xi)}{\Delta_{n}\left(\mu, h_{+}, h_{-}\right)} \operatorname{sh}\left(n h_{-}\right) \quad(0<\xi<\pi), \\
& L_{2}^{*}(\xi)=\sum_{n=1}^{\infty} \frac{\cos (n \xi)}{\Delta_{n}\left(\mu, h_{+}, h_{-}\right)} \operatorname{sh}\left(n h_{+}\right)
\end{aligned}
$$

and $\Delta_{n}\left(\mu, h_{+}, h_{-}\right)=\mu \operatorname{sh}\left(n h_{+}\right) \operatorname{ch}\left(n h_{-}\right)+\operatorname{ch}\left(n h_{+}\right) \operatorname{sh}\left(n h_{-}\right)$and the corresponding dimensionless parameters are

$$
\begin{gathered}
\xi=\frac{\pi x}{l} ; \quad \eta=\frac{\pi s}{l} ; \quad h_{+}=\frac{\pi h_{1}}{l} ; \\
h_{-}=\frac{\pi h_{2}}{l} ; \quad \mu=\frac{G_{1}}{G_{2}}, \\
\bar{\tau}_{j}(\xi)=G_{*} \tau_{j}\left(\frac{l \xi}{\pi}\right) ; \quad \tilde{\tau}_{ \pm}(\xi)=G_{*} \tau_{ \pm}^{(0)}\left(\frac{l \xi}{\pi}\right), \\
\bar{\tau}(\xi)=G_{*} \tau\left(\frac{l \xi}{\pi}\right) ; \quad G_{*}=\frac{G_{1}+G_{2}}{2 G_{1} G_{2}}, \\
\alpha_{k}=\frac{\pi a_{k}}{l} ; \quad \beta_{k}=\frac{\pi b_{k}}{l} ; \quad(k=1, \bar{N}), \\
L_{0}=\bigcup_{k=1}^{N}\left[\alpha_{k}, \beta_{k}\right] ; \quad L_{0}^{\prime}=[0, \pi] \backslash L_{0} ; \quad 0<\xi, \eta<\pi .
\end{gathered}
$$


In this manner the system of lengths $(L)$ converts to system $\left(L_{0}\right)$, and, through function (13), the dislocation density is imposed at the boundaries of the crack edges:

$$
\begin{gathered}
\phi^{\prime}(x)= \begin{cases}\varphi^{\prime}(x) & (x \in L) ; \\
0 & \left(x \in L^{\prime}\right) ;\end{cases} \\
=\frac{2}{\pi} \sum_{n=1}^{\infty} \varphi_{n} \cos (n \xi), \\
\varphi_{n}=\frac{\pi n}{l} \phi_{n}=\frac{l}{\pi} \int_{L_{0}} \varphi_{0}(\xi) \cos (n \xi) d \xi, \\
\varphi_{0}(\xi)=\varphi^{\prime}\left(\frac{l \xi}{\pi}\right) .
\end{gathered}
$$

Then, by applying key equation (15) to the system $\left(L_{0}\right)$ and introducing the new variables

$$
t=\cos \xi, \quad u=\cos \eta
$$

regarding the unknown dislocation density $\varphi_{0}(\xi)$ from (18), the singular integral equation governing the problem is obtained as follows:

$$
\begin{aligned}
& \frac{1}{\pi} \int_{\Lambda_{0}} \frac{\omega_{0}(u) d u}{u-t}+\frac{1}{\pi} \int_{\Lambda_{0}}\left[\frac{(u+t) K_{0}(t, u)}{\sqrt{1-u^{2}}}-2 K(t, u)\right] \\
& \times \omega_{0}(u) d u=f(t) \quad\left(t \in \Lambda_{0}\right), \\
& f(t)=-\frac{1}{2} \widetilde{h}_{0}^{+}(t)+\frac{\mu-1}{\mu+1} \widetilde{h}_{0}^{-}(t) \\
& +\frac{4 \mu}{\pi(1+\mu)} \int_{\Lambda_{0}} L(t, u) \widetilde{h}_{0}^{-}(u) d u \\
& +\frac{2}{\pi} \int_{-1}^{1} L_{1}(t, u) \bar{\omega}_{1}(u) d u \\
& +\frac{2 \mu}{\pi} \int_{-1}^{1} L_{2}(t, u) \bar{\omega}_{2}(u) d u \\
& \tilde{h}_{0}^{ \pm}(t)=\tilde{\tau}_{+}(\arccos t) \pm \tilde{\tau}_{-}(\arccos t), \\
& \bar{\omega}_{j}(t)=\bar{\tau}_{j}(\arccos t) \quad(j=1,2, \ldots), \\
& \omega_{0}(t)=\varphi_{0}(\arccos t), \\
& K_{0}(t, u)=\left(\sqrt{1-t^{2}}+\sqrt{1-u^{2}}\right)^{-1} \\
& \Lambda_{0}=\bigcup_{k=1}^{N}\left[\delta_{k}, \gamma_{k}\right] \\
& \delta_{k}=\cos \left(\beta_{N+1-k}\right)=\cos \left(\pi \frac{b_{N+1-k}}{l}\right) \text {, } \\
& \gamma_{k}=\cos \left(\alpha_{N+1-k}\right)=\cos \left(\pi \frac{a_{N+1-k}}{l}\right) .
\end{aligned}
$$

Based on (16), the following influencing factors are derived:

$$
\begin{aligned}
K(t, u)= & \frac{\sqrt{1-t^{2}}}{\sqrt{1-u^{2}}} \sum_{n=1}^{\infty} \frac{T_{n}(u) U_{n-1}(t)}{\Delta_{n}\left(\mu, h_{+}, h_{-}\right)} \\
& \times\left[\left(\mu \operatorname{sh}\left(n h_{+}\right) e^{-n h}+\operatorname{sh}\left(n h_{-}\right) e^{-n h}\right)\right], \\
L(t, u)= & \sqrt{1-t^{2}} \sum_{n=1}^{\infty} \frac{U_{n-1}(u) U_{n-1}(t)}{\Delta_{n}\left(\mu, h_{+}, h_{-}\right)} \operatorname{sh}\left[n\left(h_{+}-h_{-}\right)\right], \\
L_{1}(t, u)= & \sqrt{1-t^{2}} \sum_{n=1}^{\infty} \frac{U_{n-1}(u) U_{n-1}(t)}{\Delta_{n}\left(\mu, h_{+}, h_{-}\right)} \operatorname{sh}\left(n h_{-}\right), \\
L_{2}(t, u)= & \sqrt{1-t^{2}} \sum_{n=1}^{\infty} \frac{U_{n-1}(u) U_{n-1}(t)}{\Delta_{n}\left(\mu, h_{+}, h_{-}\right)} \operatorname{sh}\left(n h_{+}\right),
\end{aligned}
$$

where $T_{n}(u)$ and $U_{n-1}(t)$ are Chebyshev polynomials of the first kind and the second kind, respectively.

The singular integral equations (20) and (21) in a condition that explains the continuity of displacements at the crack tips are expressed as

$$
\int_{a_{k}}^{b_{k}} \varphi^{\prime}(s) d s=0 \quad(k=\overline{1, N})
$$

which, based on the previously discussed dimensionless variables, converts to

$$
\int_{\delta_{k}}^{\gamma_{k}} \frac{\omega_{0}(u) d u}{\sqrt{1-u^{2}}}=0 \quad(k=\overline{1, N}) .
$$

Hence after solving the singular integral equations (20) and (21) by considering condition (23) and verifying key equation (15) in the region outside of the crack system $L_{0}^{\prime}$, the antiplane shear stress $\bar{\tau}(\xi)$ formula in the dimensionless form is obtained.

\section{Derivation of the Linear System of Equations}

To solve the singular integral equations (20) and (21) under condition (23), the numerical solution for singular integral equations $[7,27,28]$, which is based on the Gauss quadratic solutions for ordinary and singular integrals, is used. First, each section of $\left[\delta_{k}, \gamma_{k}\right](k=\overline{1, N})$ from system $\Lambda_{0}$, by means of the technique of transitioning to new variables $r, \rho(-1 \leq$ $r, \rho \leq 1)$

$$
\begin{array}{r}
t=\frac{\gamma_{k}-\delta_{k}}{2} r+\frac{\gamma_{k}+\delta_{k}}{2} ; \quad u=\frac{\gamma_{k}-\delta_{k}}{2} \rho+\frac{\gamma_{k}+\delta_{k}}{2} \\
(k=\overline{1, N})
\end{array}
$$


can be converted to the section $[-1,1]$. The singular integral equations (20) and (21) can therefore be written as follows:

$$
\begin{aligned}
& \frac{1}{\pi} \int_{-1}^{1} \frac{\omega_{k}^{(0)}(\rho) d \rho}{\rho-r}+\frac{1}{\pi} \sum_{\substack{n=1 \\
(n \neq k)}}^{N} \int_{-1}^{1} L_{k n}(r, \rho) \omega_{n}^{(0)}(\rho) d \rho \\
& +\frac{1}{2 \pi} \sum_{n=1}^{N} \int_{-1}^{1} K_{k n}(r, \rho) \omega_{n}^{(0)}(\rho) d \rho=f_{k}(r), \\
& f_{k}(r)=f\left(\frac{\gamma_{k}-\delta_{k}}{2} r+\frac{\gamma_{k}+\delta_{k}}{2}\right), \\
& L_{k n}(r, \rho)=\left(\rho-\frac{\gamma_{k}-\delta_{k}}{\gamma_{n}-\delta_{n}} r+\frac{\gamma_{n}+\delta_{n}}{\gamma_{n}-\delta_{n}}-\frac{\gamma_{k}+\delta_{k}}{\gamma_{n}-\delta_{n}}\right)^{-1}, \\
& K_{k n}(r, \rho) \\
& =\left(\gamma_{n}-\delta_{n}\right) \\
& \times\left\{\frac{\left(\gamma_{n}-\delta_{n}\right) \rho+\left(\gamma_{k}-\delta_{k}\right) r+\gamma_{n}+\delta_{n}+\gamma_{k}+\delta_{k}}{\sqrt{4-\left[\left(\gamma_{n}-\delta_{n}\right) \rho+\gamma_{n}+\delta_{n}\right]^{2}}}\right. \\
& \times K_{0}\left(\frac{\gamma_{k}-\delta_{k}}{2} r+\frac{\gamma_{k}+\delta_{k}}{2}, \frac{\gamma_{n}-\delta_{n}}{2} \rho+\frac{\gamma_{n}+\delta_{n}}{2}\right) \\
& \left.-2 K\left(\frac{\gamma_{k}-\delta_{k}}{2} r+\frac{\gamma_{k}+\delta_{k}}{2}, \frac{\gamma_{n}-\delta_{n}}{2} \rho+\frac{\gamma_{n}+\delta_{n}}{2}\right)\right\} \text {, } \\
& \omega_{k}^{(0)}(r)=\omega_{0}\left(\frac{\gamma_{k}-\delta_{k}}{2} r+\frac{\gamma_{k}+\delta_{k}}{2}\right) \\
& (k=\overline{1, N},-1<r<1), \quad(-1<r, \rho<1) \text {. }
\end{aligned}
$$

The function $f(t)$ and kernel functions $K_{0}(t, u)$ and $K(t, u)$ are expressed by relations (20) and (21). In this case, condition equation (23) may be expressed in the following form:

$$
\begin{array}{r}
\int_{-1}^{1} \frac{\omega_{k}^{(0)}(\rho) d \rho}{\sqrt{4-\left[\left(\gamma_{k}-\delta_{k}\right) \rho+\left(\gamma_{k}+\delta_{k}\right)\right]^{2}}}=0 \\
(k=\overline{1, N}) .
\end{array}
$$

Using the above approach, the singular integral equation (25) through condition equation (26) is reduced to a system of linear algebraic equations:

$$
\begin{gathered}
\frac{1}{M} \sum_{p=1}^{M}\left[\frac{\psi_{k}\left(\rho_{p}\right)}{\rho_{p}-r_{m}}+\sum_{\substack{n=1 \\
(n \neq k)}}^{N} \int_{-1}^{1} L_{k n}\left(r_{m}, \rho_{p}\right) \psi_{n}\left(\rho_{p}\right)\right. \\
\left.+\frac{1}{2} \sum_{\substack{n=1 \\
(n \neq k)}}^{N} K_{k n}\left(r_{m}, \rho_{p}\right) \psi_{n}\left(\rho_{p}\right)\right]=f_{k}\left(r_{m}\right) \\
(m=\overline{1, M-1}, k=\overline{1, N}), \\
\frac{1}{M} \sum_{p=1}^{M} \frac{\psi_{k}\left(\rho_{p}\right)}{\sqrt{4-\left[\left(\gamma_{k}-\delta_{k}\right) \rho_{p}+\gamma_{k}-\delta_{k}\right]^{2}}}=0 .
\end{gathered}
$$

In (25), (26), it is supposed that

$$
\omega_{k}^{(0)}(\rho)=\frac{\psi_{k}(\rho)}{\sqrt{1-\rho^{2}}} \quad(k=\overline{1, N})
$$

where $M$ is an arbitrary natural number. Consider

$$
\begin{aligned}
& r_{m}=\cos \left(\frac{\pi m}{M}\right) \quad(m=1, M-1), \\
& \rho_{p}=\cos \left(\frac{2 p-1}{2 M} \pi\right) \quad(p=\overline{1, M})
\end{aligned}
$$

are the roots of the Chebyshev polynomials of the second kind $U_{m-1}(r)$ and of the first kind $T_{M}(\rho)$.

The opening displacement of each crack $L_{k}=\left[a_{k}, b_{k}\right]$ is given by the equations:

$$
\begin{array}{r}
\Phi(x)=\int_{a_{k}}^{x} \varphi^{\prime}(s) d s, \quad \Phi(x)=-\int_{x}^{b_{k}} \varphi^{\prime}(s) d s \\
\left(a_{k} \leq x \leq b_{k}, k=\overline{1, N}\right)
\end{array}
$$

which can be expressed by means of the dimensionless variables as

$$
\begin{aligned}
& \Phi_{N+1-k}^{(0)}(r) \\
& =-\frac{\gamma_{N+1-k}-\delta_{N+1-k}}{2 \pi} \\
& \quad \times \int_{-1}^{1} \frac{\operatorname{sgn}(r-\rho) \omega_{N+1-k}^{(0)}(\rho) d \rho}{\sqrt{4-\left[\left(\gamma_{N+1-k}-\delta_{N+1-k}\right) \rho+\gamma_{N+1-k}+\delta_{N+1-k}\right]^{2}}} \\
& \quad(-1 \leq r \leq 1, k=\overline{1, N}),
\end{aligned}
$$

where

$$
\Phi_{N+1-k}^{(0)}(r)=\Phi\left(\frac{l \xi}{\pi}\right) l^{-1}=l^{-1} \Phi\left(\frac{l}{\pi} \arccos t\right) .
$$

It must be noted that the system of equations (27) can be solved by the Gaussian technique, and (31) also can be solved with high accuracy by means of the Gauss quadratic formula using (28).

At the end, the stress intensity factors, SIFs, for the antiplane mode of fracture at the crack tips $\left[a_{k}, b_{k}\right]$ are obtained according to [24] $(k=\overline{1, N})$ :

$$
\begin{aligned}
& K_{\mathrm{III}}\left(a_{k}\right)=\frac{G_{1} G_{2}}{G_{1}+G_{2}} \lim _{x \rightarrow a_{k}+0}\left[\sqrt{2 \pi\left(x-a_{k}\right)} \varphi^{\prime}(x)\right], \\
& K_{\mathrm{III}}\left(b_{k}\right)=\frac{G_{1} G_{2}}{G_{1}+G_{2}} \lim _{x \rightarrow b_{k}+0}\left[\sqrt{2 \pi\left(b_{k}-x\right)} \varphi^{\prime}(x)\right]
\end{aligned}
$$

which can be converted to the dimensionless form and be expressed through $\psi_{k}(\rho)$ using formula (28). 
Because of the large number of variable parameters involved in the problem, only three different cases are considered.

Case A: a plate with one symmetric crack: the crack length is assumed arbitrary, and antiplane shear loading is two point loads. The stress and dislocation states are studied for different crack sizes and shear modulus ratios.

Case B: a plate with two cracks: the cracks are located symmetrically and have arbitrary length and distance. The stress and dislocation states are studied for different crack sizes, distances between adjacent crack tips, and shear modulus ratios.

Case C: it is a plate with thin-walled inclusions at the crack tips, in which the sensitivity of SIFs to the size of the inclusion domain with respect to crack length and the effect of the shear modulus ratio of the inclusion material to the plate on the stress state are studied.

\section{Case A: Plate with One Crack}

A rectangular plate in the Cartesian coordinate system Oxyz is considered, which is composed of an upper rectangle $D_{1}$ with modulus of rigidity $G_{1}$, length $l$, and height $h$ and a lower rectangle $D_{2}$ with modulus of rigidity $G_{2}$, length the same as $l$, and height $h$. On the interface surface line of the two rectangular plates in the interval $0 \leq x \leq l$, there is a central crack with length equal to $2 a$ (Figure 2 ):

$$
L=\left[a_{1}, b_{1}\right], \quad a_{1}=\frac{l}{2}-a, b_{1}=\frac{l}{2}+a\left(0<a<\frac{l}{2}\right) .
$$

The boundaries of the crack indeed have not any loadings. Furthermore, the vertical edges $x=0$ and $x=l$ of rectangles $D_{j}(j=1,2)$ are restrained, and the upper and lower horizontal edges $y= \pm h$ at the midpoints $(l / 2, \pm h)$ are stressed by antiplane shear forces equal to $P$

$$
\left.\tau_{y z}^{(1)}\right|_{y=h-0}=\left.\tau_{y z}^{(2)}\right|_{y=-h+0}=P \delta\left(x-\frac{l}{2}\right) \quad(0<x<l)
$$

in which $\tau_{y z}^{(j)}(j=1,2)$ are antiplane shear stresses on the top and bottom boundaries of plates $D_{j}$ and $\delta(x)$ is the Dirac delta function.

It is necessary to determine the dislocation field around the crack boundaries, the stress intensity factors, SIFs, and the shearing stresses on the interface surface $L^{\prime}=[0, l] \backslash L$ outside of the crack region.

The singular integral equation (SIE) governing the current problem can be found in the previous section, particularly by observing the dislocation field around the crack boundaries as follows:

$$
\begin{gathered}
\phi^{\prime}(x)= \begin{cases}\varphi^{\prime}(x) & \left(x \in\left[a_{1}, b_{1}\right]=L\right) ; \\
0 & \left(x \in L^{\prime}\right) ;\end{cases} \\
\phi(x)=\frac{1}{2}\left[w_{1}(x, 0)-w_{2}(x, 0)\right] \quad(0 \leq x \leq l)
\end{gathered}
$$

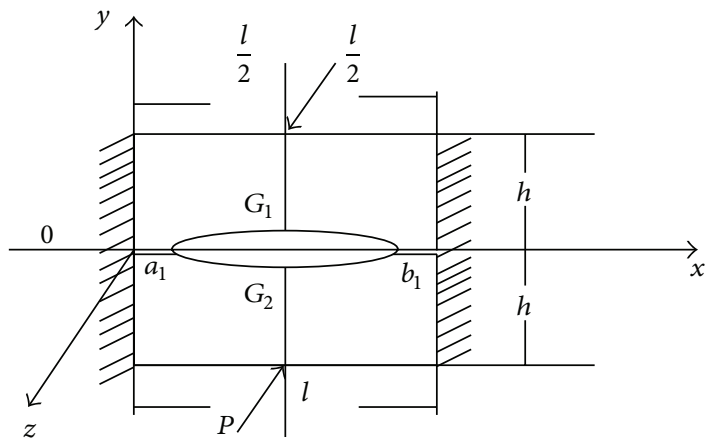

FIGURE 2: Plate with one crack.

in which $w_{j}(x, 0)$ are the displacement components of region $\{y= \pm 0,0 \leq x \leq l\}$ of rectangles $D_{j}(j=1,2)$ in the direction of $\mathrm{O} z$, so the singular integral equation of this boundary value problem from [29] is written as follows:

$$
\begin{gathered}
\frac{1}{\pi} \int_{\Lambda_{0}} \frac{\omega_{0}(u) d u}{u-t}+\frac{1}{\pi}\left[\frac{(u+t) K_{0}(t, u)}{\sqrt{1-u^{2}}}-2 K(t, u)\right] \\
\times \omega_{0}(u) d u=f(t) \quad\left(t \in \Lambda_{0}\right), \\
\omega_{0}(t)=\varphi^{\prime}\left(\frac{1}{\pi} \arccos t\right), \\
K_{0}(t, u)=\left(\sqrt{1-t^{2}}+\sqrt{1-u^{2}}\right)^{-1} \\
K(t, u)=\frac{\sqrt{1-t^{2}}}{\sqrt{1-u^{2}}} \sum_{n=1}^{\infty} \frac{T_{n}(u) U_{n-1}(t)}{\operatorname{ch}\left(n h_{0}\right)} e^{-n h}, \\
\Lambda_{0}=\left(\delta_{1}, \gamma_{1}\right) ; \quad \delta_{1}=-\sin \alpha ; \gamma_{1}=\sin \alpha \\
\alpha=\frac{\pi a}{l} ; \quad h_{0}=\frac{\pi h}{l} \\
f(t)=2 Q_{0} \sqrt{1-t^{2}} \sum_{n=1}^{\infty}(-1)^{n-1} \frac{U_{2 n-2}(t)}{\operatorname{ch}\left[(2 n-1) h_{0}\right]} \\
Q_{0}=\frac{\left(G_{1}+G_{2}\right) P}{2 G_{1} G_{2} l}
\end{gathered}
$$

where $T_{n}(t)$ and $U_{n-1}(t)$ are Chebyshev polynomials of the first kind and the second kind, respectively.

To solve the singular integral equation (37), the Gaussian quadratic solution for ordinary and singular Cauchy integrals can be used. By substitution,

$$
\omega_{1}^{(0)}(\rho)=\frac{\psi_{1}(\rho)}{\sqrt{1-\rho^{2}}} \quad(-1<\rho<1) .
$$


Using the approach shown here and also in $[7,27,28]$, solving the singular integral equation (37) leads to a system of linear algebraic equations as follows:

$$
\begin{aligned}
& \sum_{p=1}^{M} \frac{1}{M}\left[\frac{1}{\rho_{p}-r_{m}}+\frac{1}{2} K_{11}\left(r_{m}, \rho_{p}\right)\right] \psi_{1}\left(\rho_{p}\right) \\
& =f_{1}\left(r_{m}\right) \quad(m=\overline{1, M-1}) \text {, } \\
& \sum_{p=1}^{M} \frac{1}{M} \frac{\psi_{1}\left(\rho_{p}\right)}{\sqrt{1-\rho_{p}^{2} \sin ^{2} \alpha}}=0, \\
& K_{11}\left(r_{m}, \rho_{p}\right)=\left(\frac{2 \sin ^{2} \alpha\left(r_{m}+\rho_{p}\right)}{\sqrt{1-\rho_{p}^{2} \sin ^{2} \alpha}}\right. \\
& \left.\times \frac{1}{\left(\sqrt{1-r_{m}^{2} \sin ^{2} \alpha+\sqrt{1-\rho_{p}^{2} \sin ^{2} \alpha}}\right)}\right) \\
& -4 \sin \alpha \frac{\sqrt{1-r_{m}^{2} \sin ^{2} \alpha}}{\sqrt{1-\rho_{p}^{2} \sin ^{2} \alpha}} \\
& \times \sum_{n=1}^{\infty} \frac{e^{-n h_{0}}}{\operatorname{ch}\left(n h_{0}\right)} T_{n}\left(\rho_{p} \sin \alpha\right) U_{n-1}\left(r_{m} \sin \alpha\right), \\
& f_{1}\left(r_{m}\right)=2 Q_{0} \sqrt{1-r_{m}^{2} \sin ^{2} \alpha} \\
& \times \sum_{n=1}^{\infty}(-1)^{n-1} \frac{U_{2 n-2}\left(r_{m} \sin \alpha\right)}{\operatorname{ch}\left[(2 n-1) h_{0}\right]}, \\
& r_{m}=\cos \left(\frac{\pi m}{M}\right) \quad(m=\overline{1, M-1}), \\
& \rho_{p}=\cos \left(\frac{2 p-1}{2 M} \pi\right) \quad(p=\overline{1, M}),
\end{aligned}
$$

where $M$ is an arbitrary natural number and $r_{m}$ and $\rho_{p}$ are the roots of Chebyshev polynomials of the second kind $U_{M-1}(r)$ and the first kind $T_{M}(\rho)$, respectively. We can substitute the below parameters to express the system of equations (39) in a simpler form:

$$
\begin{gathered}
X_{p}=\psi_{1}\left(\rho_{p}\right) \quad(p=\overline{1, M}) ; \\
a_{m}= \begin{cases}f_{1}\left(r_{m}\right) & (m=\overline{1, M-1}) ; \\
0 & (m=M),\end{cases} \\
K_{m p}=\left\{\begin{array}{l}
\frac{1}{M}\left[\frac{1}{\rho_{p}-r_{m}}+\frac{1}{2} K_{11}\left(r_{m}, \rho_{p}\right)\right] \\
\frac{1}{M} \frac{1}{\sqrt{1-M} ; m=\overline{1, M-1})} \\
(p=1, M ; m=1, M-1) .
\end{array}\right.
\end{gathered}
$$

Hence, the system of equations (39) is reduced to

$$
\sum_{p=1}^{M} K_{m p} X_{p}=a_{m} \quad(m=\overline{1, M}) .
$$

By assumption of the below expression for the right hand side of (41)

$$
a_{m}^{(0)}=\left\{\begin{array}{lc}
\sqrt{1-r_{m}^{2} \sin ^{2} \alpha} \sum_{n=1}^{\infty}(-1)^{n-1} & \frac{U_{2 n-2}\left(r_{m} \sin \alpha\right)}{\operatorname{ch}\left[(2 n-1) h_{0}\right]} \\
0 & (m=\overline{1, M-1}) \\
0 & (m=M) .
\end{array}\right.
$$

$X_{p}^{(0)}(p=\overline{1, M})$ is found. The solution of system (26) is thereby obtained as follows:

$$
X_{p}=2 Q_{0} X_{p}^{(0)} \quad(p=\overline{1, M}) .
$$

For the derivation of SIF, the general formula from $[7,30]$ may be used; that is,

$$
\begin{aligned}
K_{\mathrm{III}} & =\lim _{x \rightarrow b_{1}+0}\left\lfloor\sqrt{2 \pi\left(x-b_{1}\right)} \tau_{y z}(x, 0)\right\rfloor \\
& =\lim _{x \rightarrow b_{1}+0}\left\lfloor\sqrt{2 \pi\left(x-b_{1}\right)} \tau(x)\right\rfloor .
\end{aligned}
$$

The above formula can be converted to a dimensionless form as follows:

$$
\begin{aligned}
K_{\mathrm{III}}= & \frac{2 G_{1} G_{2}}{G_{1}+G_{2}} \\
& \times \lim _{x \rightarrow 1+0}\left[\sqrt{2 l\left|\arccos (-r \sin \alpha)-\left(\frac{\pi}{2}+\alpha\right)\right|} \tau_{0}(t)\right] .
\end{aligned}
$$

\section{Case B: Plate with Two Cracks}

In this section, a rectangular plate in the Cartesian coordinate system $O x y z$ with two cracks is considered. On the interface surface of the two segments in the interval $0 \leq x \leq l$ there are two central cracks located symmetrically at $L=\cup_{k=1}^{2}\left[a_{k}, b_{k}\right]$ that have equal lengths (Figure 3):

$$
\begin{array}{r}
a_{1}=\frac{l}{2}-a, \quad b_{1}=\frac{l}{2}-b, \\
a_{2}=\frac{l}{2}+b, \quad b_{2}=\frac{l}{2}+a \\
\quad\left(0<a<\frac{l}{2} ; b<a\right) .
\end{array}
$$

The boundaries of the cracks have no traction; furthermore, the vertical edges of plates $D_{j}(j=1,2)$ at $x=0$ and $x=l$ are clamped, and the upper and lower horizontal edges $y= \pm h$ are loaded by antiplane distributed shear loading $T(x)$, so that

$$
\left.\tau_{y z}^{(1)}\right|_{y=h-0}=\left.\tau_{y z}^{(2)}\right|_{y=-h+0}=T(x) \quad(0<x<l),
$$




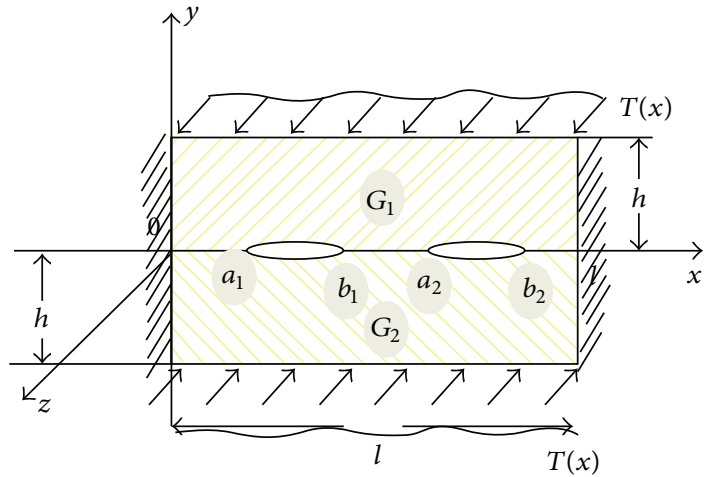

Figure 3: Plate with two cracks.

in which $\tau_{y z}^{(j)}(j=1,2)$ are the antiplane shear stresses on the top and bottom boundaries of segment $D_{j}$.

The displacement of the crack boundaries $L$, the stress intensity factors SIFs, and the shear stresses at the interface surface $L^{\prime}=[0, l] \backslash L$ outside of the cracks are determined. Moreover, it is necessary to investigate the influence of adjacent crack tips $a_{2}$ and $b_{1}$.

Suppose that the upper and lower edges of the plate are stressed by antiplane shear forces $P$ so that $T(x)=P \delta(x-$ $l / 2)$ in which $\delta(x)$ is the known Dirac delta function. By this assumption, the function $f(t)$ is calculated as follows:

$$
\begin{gathered}
f(t)=Q_{0} \sqrt{1-t^{2}} \sum_{n=1}^{\infty} \frac{(-1)^{n-1}}{\operatorname{ch}\left[(2 n-1) h_{0}\right]} U_{2 n-2}(t) \\
(-1<t<1), \\
Q_{0}=\frac{P\left(G_{1}+G_{2}\right)}{G_{1} G_{2} l} .
\end{gathered}
$$

To calculate the shear stress, making use of the variables shown before and variable $\rho$ and taking into consideration (38), the shear stress is concluded:

$$
\begin{aligned}
& \tau_{0}(t) \\
& =\left(-\frac{1}{2 \pi} \sum_{k=1}^{2}\left(\gamma_{k}-\delta_{k}\right)\right. \\
& \left.\quad \times \int_{-1}^{1} \frac{\psi_{k}^{(0)}(\rho) d \rho}{\sqrt{1-\rho^{2}}\left(\left(\left(\gamma_{k}-\delta_{k}\right) / 2\right) \rho+\left(\gamma_{k}+\delta_{k}\right) / 2-t\right)}\right) \\
& -\left(\frac{1}{2 \pi} \sum_{k=1}^{2}\left(\gamma_{k}-\delta_{k}\right)\right. \\
& \quad \times \int_{-1}^{1}\left[\frac{\left(\gamma_{k}-\delta_{k}\right) \rho+\gamma_{k}+\delta_{k}+2 t}{\sqrt{4-\left[\left(\gamma_{k}-\delta_{k}\right) \rho+\gamma_{k}+\delta_{k}\right]^{2}}}\right.
\end{aligned}
$$

$$
\begin{gathered}
\times K_{0}\left(t, \frac{\gamma_{k}-\delta_{k}}{2} \rho+\frac{\gamma_{k}+\delta_{k}}{2}\right) \\
\left.\left.-2 K\left(t, \frac{\gamma_{k}-\delta_{k}}{2} \rho+\frac{\gamma_{k}+\delta_{k}}{2}\right)\right]\right) \\
\times\left(\frac{\psi_{k}^{(0)}(\rho) d \rho}{\sqrt{1-\rho^{2}}}\right)+f(t) \quad\left(t \in \frac{[-1,1]}{\Lambda_{0}}\right) \\
\Lambda_{0}=[-\sin \alpha,-\sin \beta] \cup[\sin \beta, \sin \alpha] .
\end{gathered}
$$

From the symmetry of the problem due to axis $x=l / 2$ and taking into consideration only the right hand crack with end points at $x=a_{2}$ and $x=b_{2}$, the stress intensity factors are defined as follows $[3,4,7,26]$ :

$$
\begin{aligned}
K_{\mathrm{III}}\left(a_{2}\right) & =\lim _{x \rightarrow a_{2}-0}\left\lfloor\sqrt{2 \pi\left(a_{2}-x\right)} \tau_{y z}\right\rfloor \\
& =\lim _{x \rightarrow a_{2}-0}\left\lfloor\sqrt{2 \pi\left(a_{2}-x\right)} \tau(x)\right\rfloor, \\
K_{\mathrm{III}}\left(b_{2}\right) & =\lim _{x \rightarrow b_{2}+0}\left\lfloor\sqrt{2 \pi\left(x-b_{2}\right)} \tau_{y z}\right\rfloor \\
& =\lim _{x \rightarrow b_{2}+0}\left\lfloor\sqrt{2 \pi\left(x-b_{2}\right)} \tau(x)\right\rfloor,
\end{aligned}
$$

where $\tau(x)$ are the shear stresses according to (31).

\section{Case C: Plate with Inclusions at the Crack Tips}

In this section, attention is specifically paid to the effect of inclusions at the crack tips on the stress intensity factors (SIFs).

A prismatic elastic body $\Omega$ with a rectangular cross section in Cartesian coordinates Oxyz occupying an area $\Omega=$ $\{0 \leq x \leq \ell ;-h \leq y \leq h ;-\infty<z<\infty\}$ and possessing a shear modulus $G$ is considered. The elastic body is rigidly clamped at $x=0$ and $x=l$ and loaded by shear forces equal to $T(x)$ acting both in the positive and in negative directions of the $O z$-axis by the horizontal $y= \pm h$. Furthermore, on the symmetry plane $y=0$, it has a through-the-thickness crack in the shape of the strip $\omega=\{y=0 ; \ell / 2-a<x<\ell / 2+$ $a ;-\infty<z<\infty\},(a<\ell / 2)$. Shear forces of equal intensities $T_{0}(x)$ are acting in opposite directions along the $\mathrm{Oz}$-axis on the upper $(+)$ and lower $(-)$ areas of the crack edges

$$
\omega_{ \pm}=\left\{y= \pm 0 ; \frac{\ell}{2}-b<x<\frac{\ell}{2}+b ;-\infty<z<\infty\right\}
$$

$$
(b<a)
$$




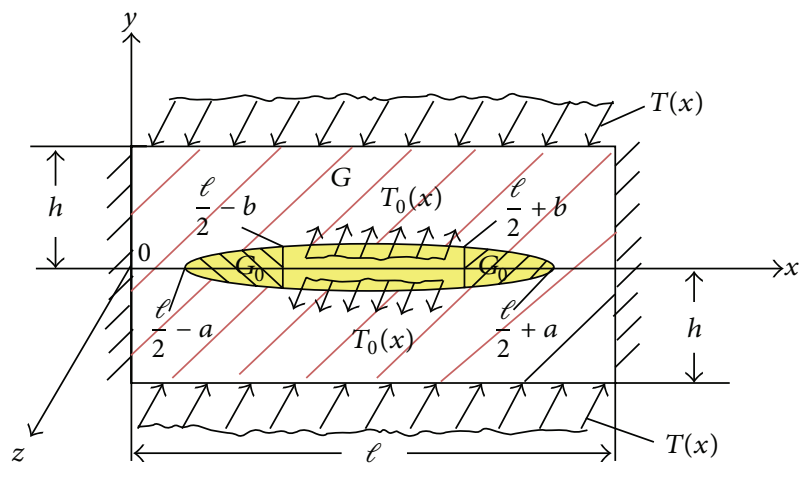

Figure 4: Plate with inclusions at crack tips.

of the crack. Additionally, at the tips

$$
\begin{aligned}
\omega_{0}^{ \pm}=\{y= \pm 0 ; x \in & \left(\frac{\ell}{2}-a ; \frac{\ell}{2}-b\right) \\
& \left.\cup\left(\frac{\ell}{2}+b ; \frac{\ell}{2}+a\right) ;-\infty<z<\infty\right\}
\end{aligned}
$$

the edges of the crack are joined by thin-walled inclusions with shear modulus $G_{0}$ deforming by the Winkler model (1867) that act as linear elastic springs: $p=k \omega$ (Figure 4) [31].

The prismatic body $\Omega$ subjected to the above-mentioned shear forces is in a state of antiplane deformation in the direction of the $O z$-axis on the basic plane $O x y$. The main rectangle $D=\{0 \leq x \leq \ell ;-h \leq y \leq h\}$ with the central $\operatorname{crack} \omega_{0}=\{y=0 ; \ell / 2-a<x<\ell / 2+a\}(0<a<\ell / 2)$ is a cross section of the body $\Omega$ at the plane $O x y$.

It is necessary to determine the dislocation density on the crack edges, the SIFs, the shear contact stresses on the edges of the inclusion, and the shear stresses outside the crack along the surface of its location.

This is formally similar to the Dugdale-Barenblatt model for a central crack containing yielding as confined and localized narrow plastic zones, which shows the effect of yielding on the crack length $[32,33]$, but, in this problem, which is a linear elastic fracture mechanics problem, we investigate the effect of inclusions at the end areas of crack on the mechanical behavior of crack tips, decreasing the dislocation density and antiplane SIFs.

The component $u_{z}=w(x, y)$ in the direction of the $O x$-axis is the only nonzero component of the displacement, and $\tau_{x z}$ and $\tau_{y z}$ are the only nonzero stress components. Therefore, the problem can be mathematically stated as a boundary value problem in the following way:

$$
\begin{aligned}
& \frac{\partial^{2} w}{\partial x^{2}}+\frac{\partial^{2} w}{\partial y^{2}}=0 \quad\left((x, y) \in D \backslash \omega_{0}\right), \\
& \left.w(x, y)\right|_{x=0}=\left.w(x, y)\right|_{x=\ell}=0 \quad(-h<y<h) ; \\
& \left.\tau_{y z}\right|_{y=h-0}=\left.\tau_{y z}\right|_{y=-h+0}=\left.G \frac{\partial w}{\partial y}\right|_{y= \pm h \mp 0}=T(x)
\end{aligned}
$$$$
(0<x<\ell) \text {; }
$$

$$
\begin{aligned}
& \left.\tau_{y z}\right|_{y= \pm 0}=\left.G \frac{\partial w}{\partial y}\right|_{y= \pm 0}=T_{0}(x) \\
& \left(x \in\left(\frac{\ell}{2}-b ; \frac{\ell}{2}+b\right) ; b<a\right) ; \\
& \left.\tau_{y z}\right|_{y= \pm 0}= \pm\left. G_{0} w(x, y)\right|_{y= \pm 0}= \pm\left. k G w(x, y)\right|_{y= \pm 0} \\
& \left(x \in\left(\frac{\ell}{2}-a ; \frac{\ell}{2}-b\right) \cup\left(\frac{\ell}{2}+b ; \frac{\ell}{2}+a\right) ; k=\frac{G_{0}}{G}\right) .
\end{aligned}
$$

Let us again consider the following functions based on [18, 34]:

$$
\begin{gathered}
\Phi(x)=\frac{w_{+}(x, 0)-w_{-}(x, 0)}{2}=\frac{2}{\ell} \sum_{n=1}^{\infty} \Phi_{n} \sin \left(\frac{\pi n x}{\ell}\right), \\
\Psi(x)=\frac{w_{+}(x, 0)+w_{-}(x, 0)}{2}=\frac{2}{\ell} \sum_{n=1}^{\infty} \Psi_{n} \sin \left(\frac{\pi n x}{\ell}\right), \\
\Omega(x)=\frac{\tau_{+}(x)+\tau_{-}(x)}{2}=\frac{2}{\ell} \sum_{n=1}^{\infty} \Omega_{n} \sin \left(\frac{\pi n x}{\ell}\right) \\
X(x)=\frac{\tau_{+}(x)-\tau_{-}(x)}{2}=\frac{2}{\ell} \sum_{n=1}^{\infty} X_{n} \sin \left(\frac{\pi n x}{\ell}\right),
\end{gathered}
$$

where $\Phi_{n}, \Psi_{n}, \Omega_{n}$, and $X_{n}$ are sine Fourier series coefficients as before. Inserting these functions into the displacements and stresses on the edges of the crack at the interval $[0, l]$ of rectangle $D_{2}$ then according to (4), the following equations are obtained:

$$
\begin{gathered}
\Phi(x)=w_{+}(x, 0) ; \quad \Psi(x) \equiv 0 ; \quad \Omega(x)=\tau_{+}(x), \\
X(x) \equiv 0 ; \quad \Phi_{n}=\bar{w}_{+}(n, 0) ; \quad \Psi_{n}=0, \\
\Omega_{n}=\bar{\tau}_{+}(n) ; \quad X_{n}=0 \quad(n=1,2, \ldots) .
\end{gathered}
$$

After some simple transformations and calculations, the following equations can be derived:

$$
\begin{aligned}
& \frac{1}{\pi} \int_{-1}^{1} \frac{\omega_{0}(\rho) d \rho}{\rho-r}+\frac{1}{\pi} \int_{-1}^{1} K_{0}(r, \rho) \omega_{0}(\rho) d \rho \\
& -\frac{1}{\pi} \int_{-1}^{1} K(r, \rho) \omega_{0}(\rho) d \rho \\
& \quad= \begin{cases}f(r)-g(r) & (r \in(-c ; c)) \\
f(r)-\lambda_{0} \psi_{0}(r) & (r \in(-1,-c) \cup(c, 1))\end{cases} \\
& \quad\left(c=\frac{\sin \beta}{\sin \alpha}\right)
\end{aligned}
$$


with

$$
\begin{gathered}
\omega_{0}(r)=\varphi_{0}(\arccos (r \cdot \sin \alpha)), \\
f(r)=\widetilde{f}(\arccos (r \cdot \sin \alpha)), \\
g(r)=\widetilde{T}_{0}(\arccos (r \cdot \sin \alpha)), \\
\psi_{0}(r)=\widetilde{\varphi}_{0}(\arccos (r \cdot \sin \alpha)), \\
f(r)=\frac{2}{\pi} \sqrt{1-\sin ^{2} \alpha \cdot r^{2}} \\
\times \int_{-1}^{1}\left[\sum_{n=1}^{\infty} \frac{U_{n-1}(r \cdot \sin \alpha) U_{n-1}(u)}{\operatorname{ch}\left(n h_{0}\right)}\right] \widetilde{T}(\arccos u) d u \\
K_{0}(r, \rho)=\frac{(r+\rho) \sin ^{2} \alpha}{\sqrt{1-\sin ^{2} \alpha \cdot \rho^{2}}} \quad 1 \\
\times \frac{(-1<r, \rho<1) .}{\left(\sqrt{1-\sin ^{2} \alpha \cdot r^{2}}+\sqrt{1-\sin ^{2} \alpha \cdot \rho^{2}}\right)}
\end{gathered}
$$

Transforming the above equation, the following equation is derived:

$$
\begin{aligned}
& \widetilde{\tau}_{0}(t)= \tau_{0}(\arccos t) \\
&=-\frac{\sin \alpha}{\pi} \int_{-1}^{1} \frac{\omega_{0}(\rho) d \rho}{\sin \alpha \cdot \rho-t}-\frac{\sin \alpha}{\pi} \\
& \times \int_{-1}^{1} \frac{(t+\rho \sin \alpha) \omega_{0}(\rho) d \rho}{\sqrt{1-\rho^{2} \cdot \sin ^{2} \alpha}\left(\sqrt{1-t^{2}}+\sqrt{1-\rho^{2} \cdot \sin ^{2} \alpha}\right)} \\
&+\frac{2 \sin \alpha}{\pi} \sqrt{1-t^{2}} \\
& \cdot \int_{-1}^{1}\left[\sum_{n=1}^{\infty} \frac{e^{-n h_{0}}}{\operatorname{ch}\left(n h_{0}\right)} U_{n-1}(t) T_{n}(\rho \cdot \sin \alpha)\right] \\
& \times \frac{\omega_{0}(\rho) d \rho}{\sqrt{1-\rho^{2} \sin ^{2} \alpha}+\frac{2}{\pi} \sqrt{1-t^{2}}} \\
& \times \int_{-1}^{1}\left[\sum_{n=1}^{\infty} \frac{U_{n-1}(t) U_{n-1}(u)}{\operatorname{ch}\left(n h_{0}\right)}\right] \widetilde{T}(\arccos u) d u \\
&(t \in(-1 ;-\sin \alpha) \cup(\sin \alpha ; 1)) .
\end{aligned}
$$

As mentioned above, the determinative singular integral equation (SIE) can be reduced to a system of linear equations. For this purpose, applying the Heaviside function

$$
H(r)= \begin{cases}1 & (r>0) \\ 0 & (r<0)\end{cases}
$$

this equation can be expressed in the following way:

$$
\begin{aligned}
\frac{1}{\pi} \int_{-1}^{1}\left\{\frac{1}{\rho-r}+K_{0}(r, \rho)-K(r, \rho)\right. \\
\left.+\frac{\lambda \sin \alpha}{2} \frac{[H(c+r)-H(c-r)] \operatorname{sign} r}{\sqrt{1-\rho^{2} \cdot \sin ^{2} \alpha}} \operatorname{sign}(r-\rho)\right\} \\
\times \omega_{0}(\rho) d \rho=f(r)-[H(r+c)-H(r-c)] g(r) \\
\left(-1<r<1 ; \lambda=\pi \lambda_{0}=k \ell\right)
\end{aligned}
$$

with the notations explained above.

Supposing

$$
\omega_{0}(\rho)=\frac{X_{0}(\rho)}{\sqrt{1-\rho^{2}}} \quad(-1<\rho<1)
$$

the determinative SIE (39) with conditions of (25) and (40) is reduced to the system of linear equations

$$
\sum_{p=1}^{M} K_{m p} X_{p}=a_{m} \quad(m=\overline{1, M}),
$$

$K_{m p}$

$$
=\left\{\begin{array}{cc}
\frac{1}{M} \frac{1}{\rho_{p}-r_{m}}+K_{0}\left(r_{m}, \rho_{p}\right)-K\left(r_{m}, \rho_{p}\right) \\
\quad+\frac{\lambda \sin \alpha \operatorname{sign} r_{m}}{2 \sqrt{1-\rho_{p}^{2} \sin ^{2} \alpha}}\left[H\left(c+r_{m}\right)-H\left(c-r_{m}\right)\right] \\
\quad \times \operatorname{sign}\left(r_{m}-\rho_{p}\right) & (m=\overline{1, M-1} ; p=\overline{1, M}) \\
\frac{1}{\sqrt{1-\rho_{p}^{2} \cdot \sin ^{2} \alpha}} & (m=M ; p=\overline{1, M}),
\end{array}\right.
$$

$a_{m}$

$$
=\left\{\begin{aligned}
f\left(r_{m}\right)-\left[H\left(r_{m}+c\right)-H\left(r_{m}-c\right)\right] g\left(r_{m}\right) \\
(m=\overline{1, M-1}) \\
0, \quad(m=M), \\
X_{p}=X_{0}\left(\rho_{p}\right) \quad(p=\overline{1, M}) ; \\
\rho_{p}=\cos \left(\frac{2 p-1}{2 M} \pi\right) \quad(p=\overline{1, M}) ; \\
r_{m}=\cos \left(\frac{\pi m}{M}\right) \quad(m=\overline{1, M-1}) .
\end{aligned}\right.
$$

For this case, the crack edges are free of shearing forces, and concentrated shearing forces are acting on the horizontal sides of the rectangular plate; that is,

$$
T_{0}(x) \equiv 0, \quad T(x)=P \delta\left(x-\frac{\ell}{2}\right),
$$


TABLE 1: Dimensionless SIF $K_{\mathrm{III}}^{(0)}$ as a function of $M$ and $\alpha$.

\begin{tabular}{lccccc}
\hline & $\alpha=\pi / 32$ & $\alpha=\pi / 16$ & $\alpha=\pi / 8$ & $\alpha=\pi / 4$ & \\
\hline$M=10$ & 1.12782 & 1.69511 & 2.44264 & 3.80191 & $5=\pi / 3$ \\
$M=20$ & 1.42096 & 2.01939 & 2.91422 & 4.52924 & 5.96371 \\
$M=30$ & 1.67019 & 2.37358 & 3.42536 & 5.32364 & 7.00971 \\
$M=40$ & 2.29343 & 3.25929 & 4.70355 & 7.31018 & 9.62542 \\
$M=50$ & 2.30110 & 3.26362 & 4.70979 & 7.31988 & 9.63819 \\
\hline
\end{tabular}

where $\delta(x)$ is a certain Dirac delta function. Additionally, the following equation can be obtained:

$$
\begin{aligned}
\bar{T}(n) & =\int_{0}^{\ell} P \delta\left(x-\frac{\ell}{2}\right) \sin \left(\frac{\pi n x}{\ell}\right) d x \\
& =P \sin \left(\frac{\pi n}{2}\right)= \begin{cases}0, & \text { npu } n=2 q ; \\
(-1)^{q+1} P, & \text { npu } n=2 q-1 \\
& (q=1,2, \ldots) .\end{cases}
\end{aligned}
$$

Taking into consideration the above-mentioned definition, the function $\tilde{f}(\xi)$ can be expressed in the following way:

$$
\begin{array}{r}
\tilde{f}(\xi)=2 P_{0} \sum_{n=1}^{\infty}(-1)^{n+1} \frac{\sin [(2 n-1) \xi]}{\operatorname{ch}\left[(2 n-1) h_{0}\right]}, \\
P_{0}=\frac{P}{\ell G} \quad(0<\xi<\pi)
\end{array}
$$

and the function $f(r)$ from (57) is obtained:

$$
\begin{aligned}
f(r)= & \tilde{f}(\arccos (r \cdot \sin \alpha)) \\
= & 2 P_{0} \sqrt{1-r^{2} \cdot \sin ^{2} \alpha} \\
& \cdot \sum_{n=1}^{\infty}(-1)^{n+1} \frac{U_{2 n-2}(r \cdot \sin \alpha)}{\operatorname{ch~}\left[(2 n-1) h_{0}\right]} \\
& (-1<r<1) .
\end{aligned}
$$

It is obvious that the function $\omega_{0}(r)$ with respect to the symmetry of line $x=l / 2$ in this special case and, consequently, the function $X_{0}(r)$ according to (61) are odd functions. Therefore, the components of the second integrals in (56) and (58) containing polynomial $r$ or $t$ in arguments tend to zero, so that the above-mentioned equations and the kernel-matrix $K_{m p}$ of the system of equations (62) are simplified.

It must be emphasized that the expressions of functions $\widetilde{f}(\xi)$ and $f(r)$ from (65), (66) on various intervals are used in the equations.

The numerical analysis of the main characteristics of the stated problem can be carried out for the considered special case.

\section{Numerical Results and Discussions}

To solve the system of equations (40) that is summarized in system (41) with the parameters shown in (42),

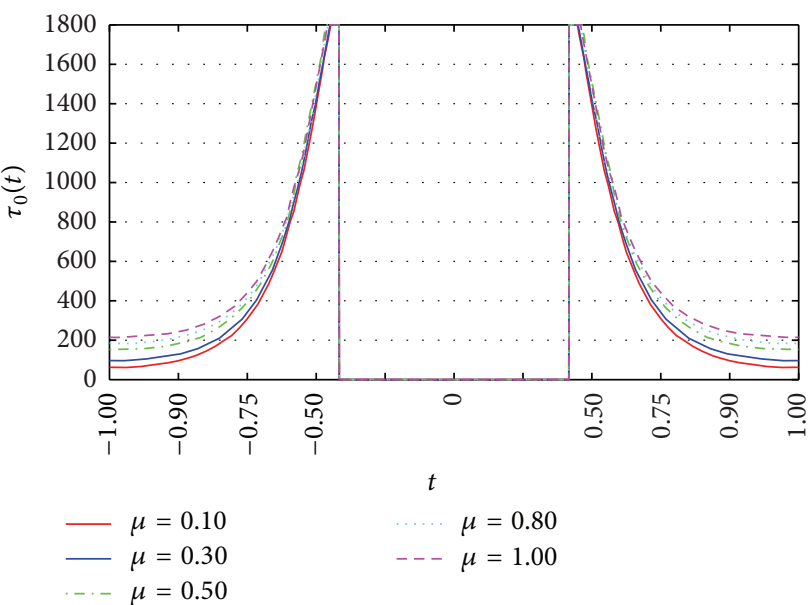

FIGURE 5: $\tau_{0}(t)$ curve for $\alpha=\pi / 4, h_{0}=0.1 \pi$, and $Q_{0}=0.01$.

$M=10,20,30,40,50$, factor $\alpha$ (which represents the cracking length) equal to $\pi / 4, h_{0}=0.1 \pi$, and $Q_{0}=0.01$ can be used. By means of Chebyshev polynomials, the deformation parameter $X_{p}$ is obtained from (43). For example, $M=10$ leads to solving $(10 * 10)$ matrix equation $(26)$.

The dimensionless SIF $K_{\mathrm{III}}^{(0)}$ according to formula (50) using the vector $X_{p}, M=10$ and considering the assumptions $\alpha=\pi / 3, \pi / 4, \pi / 8, \pi / 16$, and $\pi / 32$ for different crack lengths is calculated. The results are shown in Table 1 , indicating that longer cracks lead to larger SIFs.

The shear stress $\tau_{0}(t)$ from (49) in dependence of $t$ and $\mu$ taking the rigidity ratios of the two materials $\mu=G_{1} / G_{2}=$ $0.1,0.3,0.5,0.8,1.0,2,5,10$ is obtained and the graphs are shown in Figure 5. The dimensionless crack dislocations that depend on parameters $\alpha$ and $r$ are calculated, and the curves are represented in Figure 6. This figure shows that the maximum dislocation of a crack is at midpoint of its length, and it has a zero value at the crack tips, the points where maximum shear occurs.

To solve the system of linear equations (27) for the problem with two cracks we use iteration $M=10,15,20,30, \ldots$ in order that the results converge to the order $10^{-4}$. Calculation is based on function (27) $f_{k}(r), L_{k n}(r, \rho), K_{k n}(r, \rho)$, and $\delta_{k}, \gamma_{k}$ and (25), (37), and (39).

Parameters $\alpha$ and $\beta$ are supposed to be equal to $\alpha=\pi / 3$ and $\beta=\pi / 4 ; \pi / 8 ; \pi / 16 ; \pi / 32 ; \pi / 64$ in order to determine the influence of the distance between the two adjacent cracks on their fracture characteristics. The shear stress $\tau_{0}(t)$ is calculated according to (49), in which function $f(t)$ is obtained 


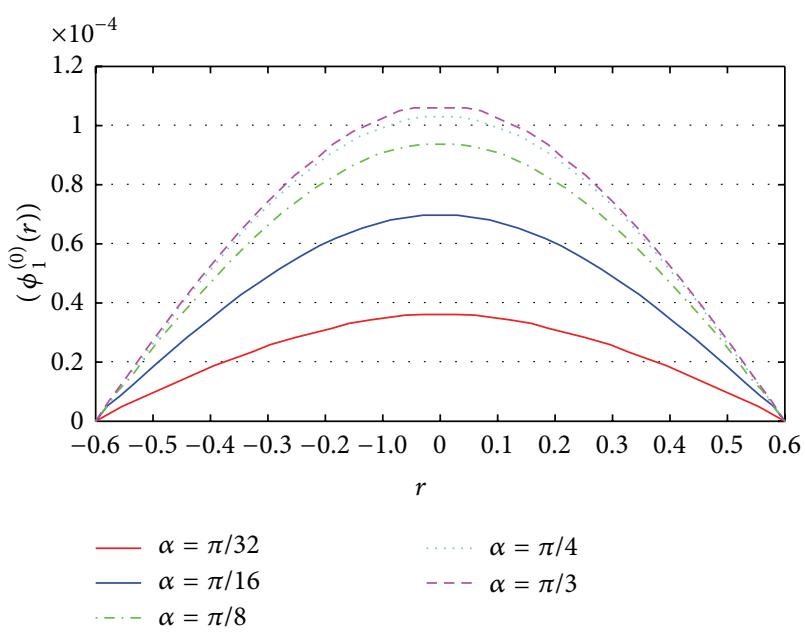

FIGURE 6: Crack dislocation $\left(\phi_{1}^{(0)}(r)\right)$ for $M=50, h_{0}=0.1 \pi$, and $Q_{0}=0.01$.

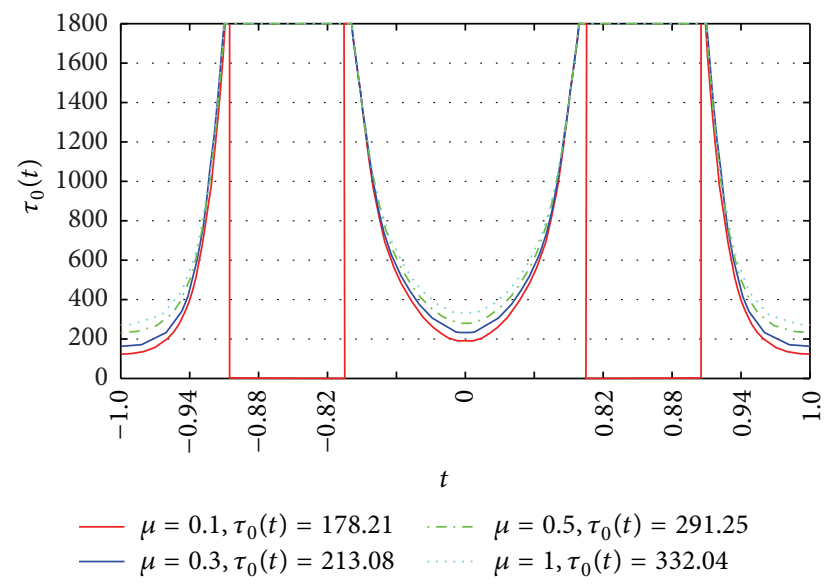

FIGURE 7: Variation of antiplane shear stress $\tau_{0}(t)$ for $\alpha=\pi / 3, \beta=$ $\pi / 4$.

from (37), supposing the quantity $Q_{0}=0.01$. Making use of the above calculated stresses, the variation curves of $\tau_{0}(t)$ can be drawn for $\mu=0.1,0.3,0.5$, and 1.0 , as shown in Figure 7 .

The crack dislocation density as a function of the edges' opening displacements $\Psi_{k}^{(0)}(r)$ may be found through formula (31). The $\Psi_{k}^{(0)}(r)$ curves are shown in Figure 8 again for varying $\mu$ and $\beta$. At the end, using (50), the stress intensity factors in the dimensionless form can easily be calculated, as shown in Figure 9 and Tables 2 and 3, for the above parameters. The variation of SIF $K_{\text {III }}$ based on parameter $\beta$ and $\mu=0.3,0.5$, and 1.0 is shown in Figure 10 to recognize the state of the SIFs under the change of the lengths of cracks.

For the case of $\mu=1.0$, the cracks approaching each other (that means a smaller value of $\beta$ ) lead us to construct curves as shown in Figure 11 for $\tau_{0}(t)$ and Figure 12 for $\Psi_{k}^{(0)}(r)$.

The main characteristics of the crack problem with inclusions in the mentioned special case can be calculated through numerical analysis. For calculation it is considered as some

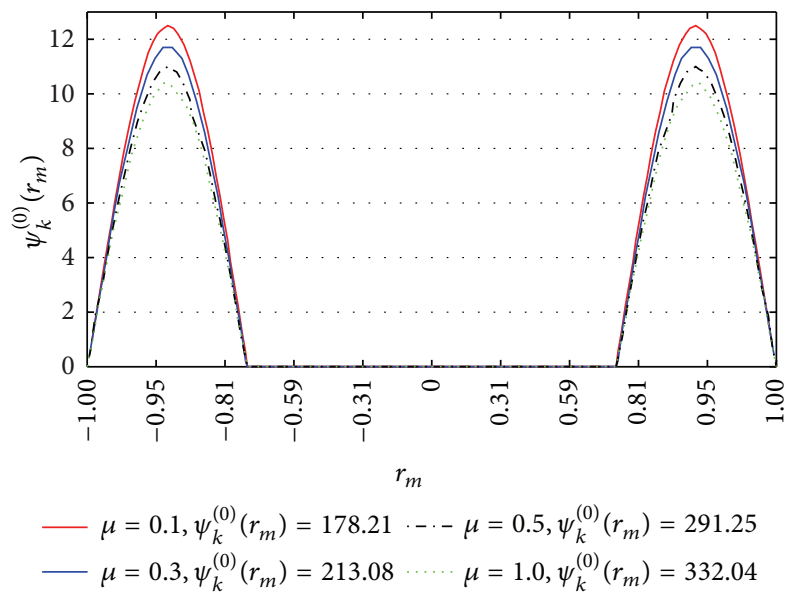

FIGURE 8: Dislocation density curves of cracks boundaries $\psi_{k}^{(0)}\left(r_{m}\right)$ for $\alpha=\pi / 3, \beta=\pi / 4$.

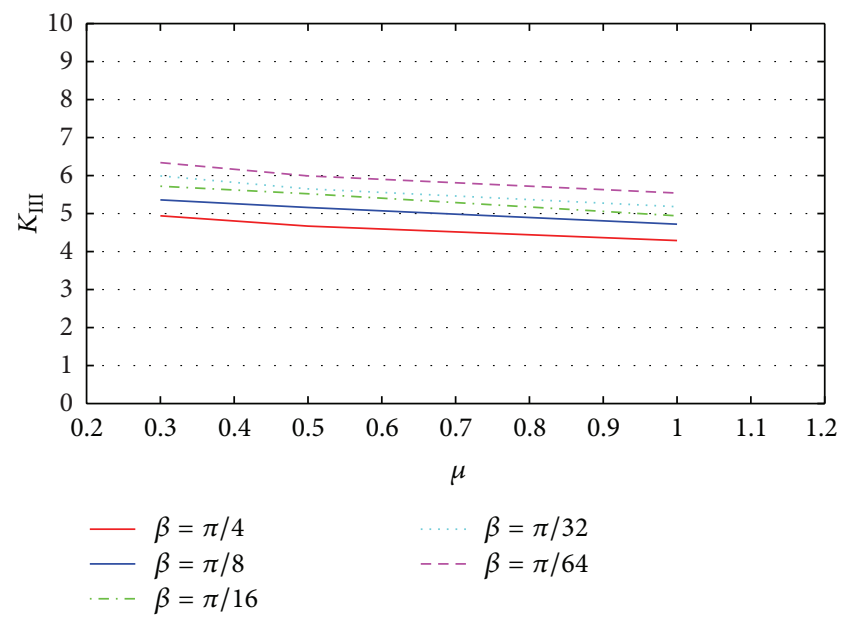

FIgURE 9: Variation of $K_{\text {III }}$ for $\alpha=\pi / 3$ based on $\beta, \mu$.

TABLE 2: Variation of $K_{\mathrm{III}}$ for $\alpha=\pi / 3$ based on $\beta, \mu$.

\begin{tabular}{lccccc}
\hline$\mu$ & & \multicolumn{5}{c}{$\beta$} & $\pi / 32$ & $\pi / 64$ \\
\hline 0.1 & 7.70 & 7.35 & 7.11 & 6.69 & 6.41 \\
0.3 & 5.36 & 5.54 & 5.73 & 6.17 & 6.35 \\
0.5 & 4.88 & 5.12 & 5.34 & 5.87 & 6.13 \\
1 & 4.21 & 4.35 & 4.63 & 5.41 & 5.92 \\
\hline
\end{tabular}

TABLE 3: Variation of $K_{\mathrm{III}}^{(0)}$ for $\alpha=\pi / 3$ based on $\beta, \lambda$.

\begin{tabular}{lcccccc}
\hline$\lambda$ & \multicolumn{5}{c}{$\beta$} & \\
& 0 & $\pi / 64$ & $\pi / 32$ & $\pi / 16$ & $\pi / 8$ & $\pi / 4$ \\
\hline 0.0 & 3.92 & 3.96 & 4.01 & 4.12 & 4.19 & 4.26 \\
0.1 & 2.87 & 3.23 & 3.69 & 3.80 & 3.98 & 4.13 \\
0.5 & 2.79 & 2.95 & 3.40 & 3.64 & 3.82 & 3.94 \\
1.0 & 2.54 & 2.72 & 3.08 & 3.37 & 3.69 & 3.82 \\
5.0 & 2.42 & 2.60 & 2.81 & 3.10 & 3.46 & 3.60 \\
20 & 2.17 & 2.34 & 2.63 & 2.88 & 3.18 & 3.45 \\
\hline
\end{tabular}




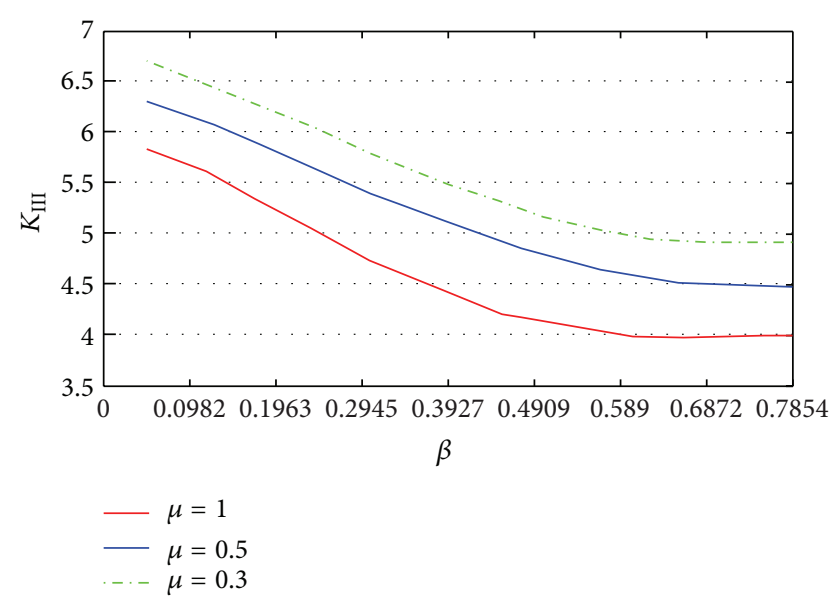

Figure 10: Variation of $K_{\text {III }}$ based on $\beta$ for $\alpha=\pi / 3$ and $\mu=$ $1,0.5,0.3$.

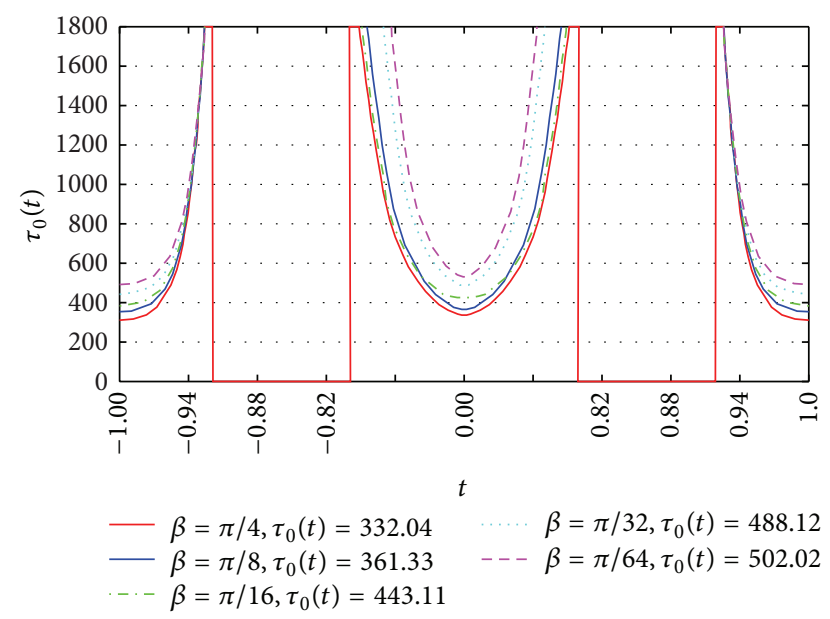

FIGURE 11: Variation of antiplane shear stress $\tau_{0}(t)$ for $\alpha=\pi / 3, \mu=1$.

engineering practical ratios for the shear modulus quantities $k=G_{0} / G$ that are $k=0.0$ (crack without repair), $k=0.5,1.0$ (repair with the same material), and $k=2.0,5.0$, and 10.0 (for very high rigid repair materials). By solving system of linear equations $(62)$ which leads to solving a matrix $(10 \times 10)$ and obtaining vectors $X_{p}(10 \times 1)$, the shear stress $\tau_{0}(t)$ can be calculated from (58) and the results are shown in Figure 13.

Dislocation functions $\widetilde{\psi}_{0}(r)=2 \varphi_{0}(r)$ were investigated using relation (31), as shown in Figure 14 in which the maximum displacement of crack boundaries occurred at midpoint and obviously with zero value at crack tips.

The stress intensity factors SIFs $K_{\mathrm{III}}^{(0)}$ calculated through (50) are shown in Figure 17, which presents the decreasing trend of the $K_{\text {III }}$ curve when the ratio of $k=G_{0} / G$ increases. It also shows that the crack tip repairing by this method can reduce the SIF by approximately 50 percent, which means that this approach is very effective to control the crack propagations in cracked plates and it is also a treatment for singularities at the crack tips, defects, and holes. Figures 15 and 16 show that the reduction of the magnitude $K_{\text {III }}$ is not

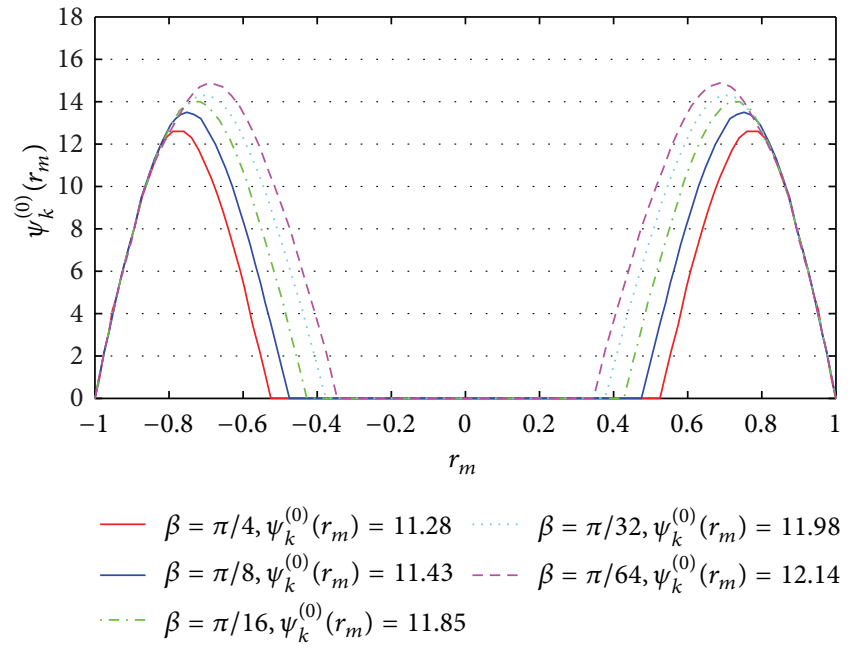

FIGURE 12: Dislocation density curves of cracks boundaries $\psi_{k}^{(0)}\left(r_{m}\right)$ for $\alpha=\pi / 3, \mu=1$.

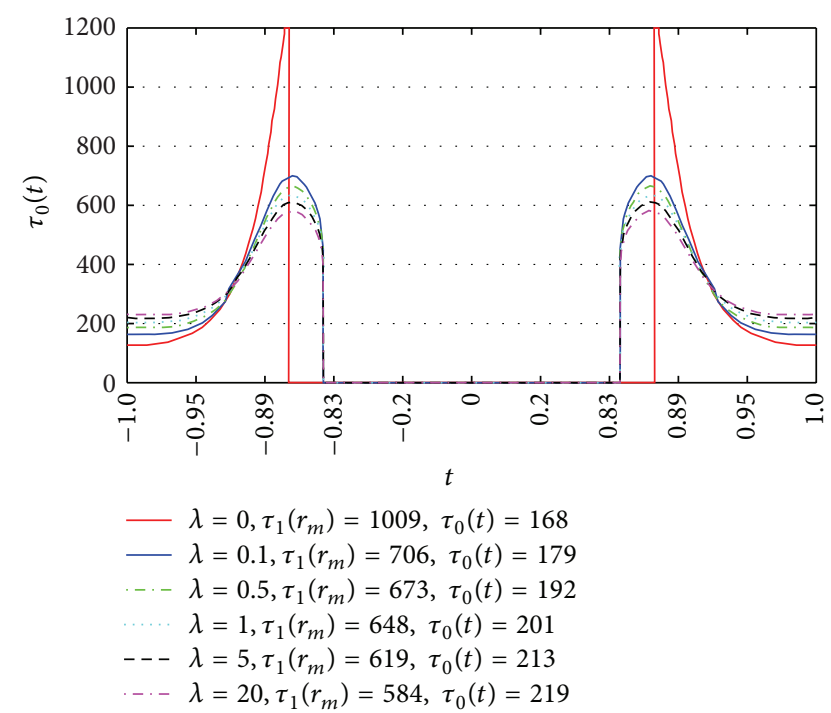

FIGURE 13: Variation of antiplane shear stress $\tilde{\tau}_{0}(t), \bar{\tau}_{1}\left(r_{m}\right)$ for $\alpha=$ $\pi / 3, \beta=\pi / 64$.

high when we use a very rigid material for tip repair. For example, for $k=2.0$ and $k=10.0($ ratio $=5)$, the reduction of $K_{\text {III }}$ is only 18 percent $(1.98 / 2.42)$.

The above results coincide with experimental observations of high concentrations and singularities in the stress fields within elastic materials $[17,18,35,36]$.

\section{Conclusion}

In the present paper, the singular integral equations governing the piecewise homogeneous elastic plate problem subject to uniform remote antiplane shear loading have been considered. To determine the antiplane shear stresses, the crack edge dislocation densities, and the mode III stress intensity factors, a new mathematical-numerical calculation 


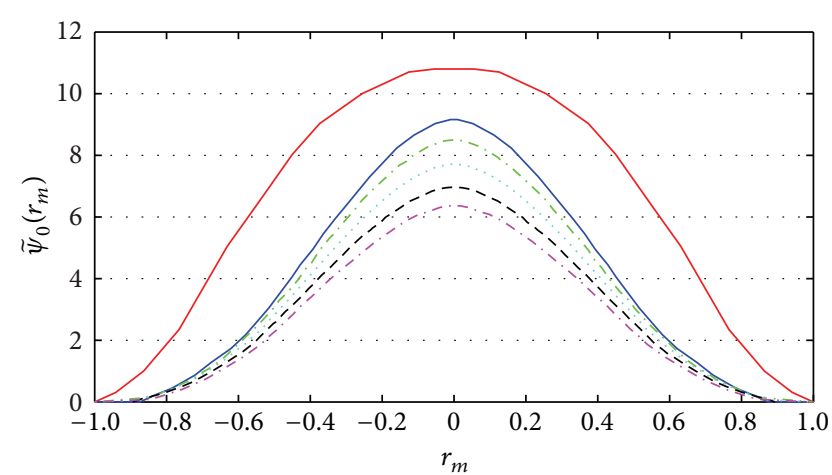

$$
\begin{aligned}
\lambda & =0, \widetilde{\psi}_{0}\left(r_{m}\right)=11.12 \quad \cdots \cdots \lambda=1, \widetilde{\psi}_{0}\left(r_{m}\right)=7.78 \\
-\lambda & =0.1, \widetilde{\psi}_{0}\left(r_{m}\right)=9.37 \quad--\lambda=5, \widetilde{\psi}_{0}\left(r_{m}\right)=6.66 \\
\lambda & =0.5, \widetilde{\psi}_{0}\left(r_{m}\right)=8.5 \quad \cdots-\lambda=20, \widetilde{\psi}_{0}\left(r_{m}\right)=6.28
\end{aligned}
$$

FIGURE 14: Dislocation curve of crack boundaries $\widetilde{\psi}_{0}\left(r_{m}\right)$ for $\alpha=$ $\pi / 3, \beta=\pi / 64$.

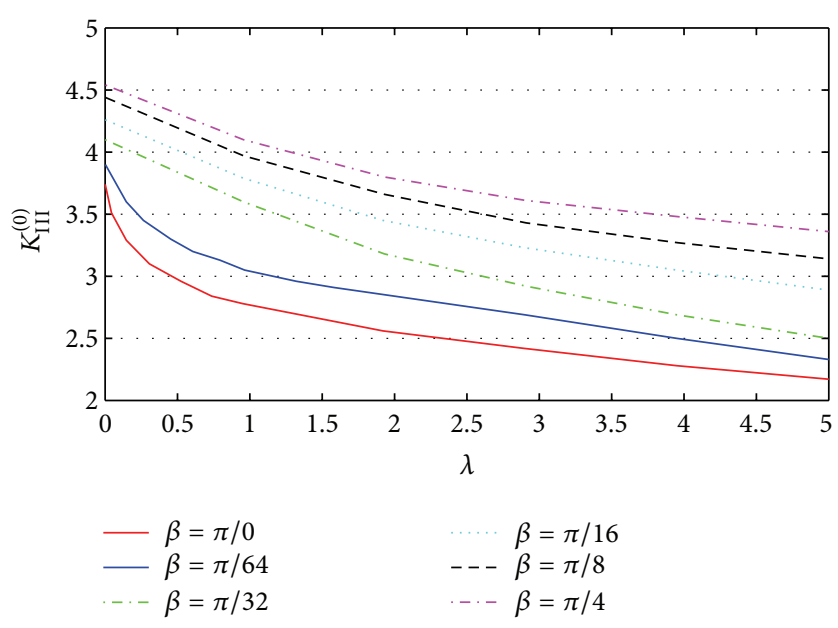

FIGURE 15: Variation of $K_{\mathrm{III}}^{(0)}$ for $\alpha=\pi / 3$ based on $\beta, \lambda$.

is developed. To perform elastic analyses and investigations on the state of stresses at the crack tips, the dimensions of the cracks, the distances between adjacent tips, and the influence of shear modulus ratios between the two materials have been varied and studied. The governing singular integral equation of a problem of stress-strain state of a piecewise homogeneous elastic prismatic body of a rectangular cross section, when there is a system of an arbitrary finite number of collinear cracks on the interface line of dissimilar materials, is obtained. For problem solving, the method of mechanical quadrature is used. It allowed conducting a detailed analysis of the main mechanical characteristics of the stated problem and revealed their dependence on the mechanical and geometrical parameters of the problem. For the case of cracks coming together it has been shown that the stress intensity factors $K_{\text {III }}$ grow based on two parameters, the total distance between their far tips and the closeness of their near tips. The technique presented in this paper can be used to solve a class of problems associated with cracking in bimaterial interfaces.

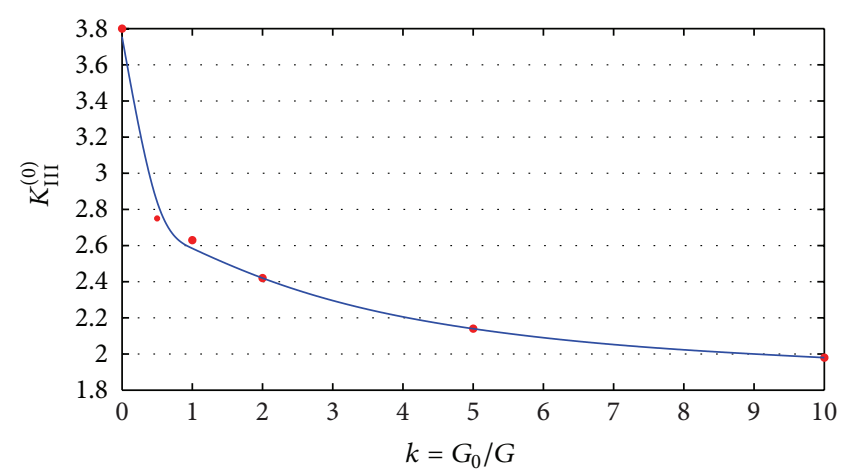

FIgURE 16: Curve of decreasing $K_{\text {III }}^{(0)}$.

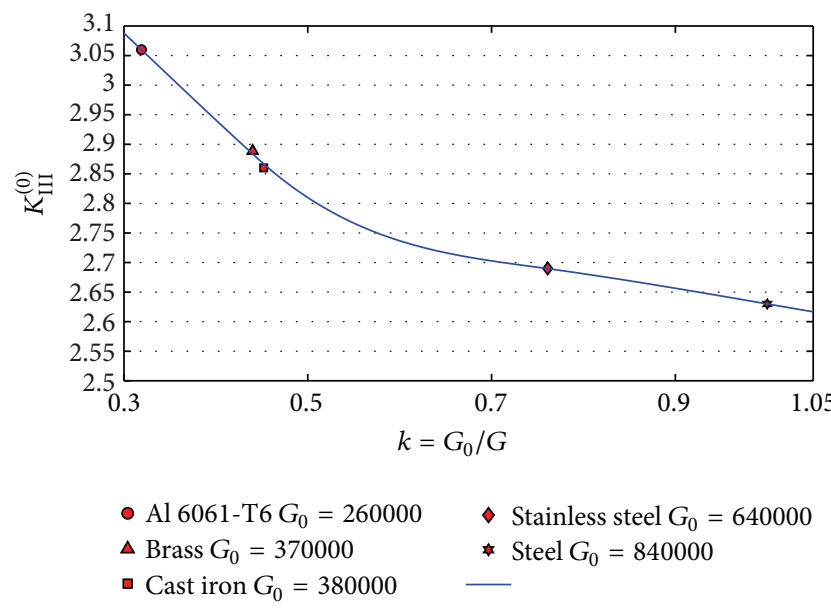

FIGURE 17: Curve of decreasing $K_{\mathrm{III}}^{(0)}$ for various inclusion materials on base metal steel with $G=840000 \mathrm{Kg} / \mathrm{cm}^{2}$.

For a crack with inclusions at the tips, it is observed that the insertion of a material at the crack tips avoids singularities, reduces the antiplane SIF $K_{\mathrm{III}}$, and strengthens and controls the crack propagation in the region at the tips. This is an effective method and does not require using a material with a very high shear rigidity value $G_{0}$. Meanwhile, the crack opening displacement (COD) and the shear stresses at the crack tips decrease. This suggests a method to repair cracked plates and members.

Practice shows that the method of mechanical quadrature is a very effective method and results in a very good convergence. The data of Table 2 confirms this. The accuracy of the method in predicting the intensity factor may be verified by a comparison with experimental measurements, carried out by a photoelasticity method, and by commercial finite element software. The drawn conclusions provide meaningful reference for the analysis of SIFs in mode III. Numerical results show the influence of ratios of shear moduli on the stress state.

The results of this paper and the previously published results show that the used approach based on the GaussChebyshev quadrature method can be considered as a generalized procedure to solve the collinear crack problems in mode I, II, or III loadings. 


\section{Appendix}

Consider

$$
\begin{array}{r}
\left(\begin{array}{c}
\Phi_{n} \\
\Psi_{n} \\
\Omega_{n} \\
\chi_{n}
\end{array}\right)=\int_{0}^{l}\left(\begin{array}{c}
\Phi(x) \\
\Psi(x) \\
\Omega(x) \\
\chi(x)
\end{array}\right) \sin \frac{\pi n x}{l} d x \\
(n=1,2, \ldots) .
\end{array}
$$

Obviously $\Phi_{n}, \Psi_{n}, \Omega_{n}$, and $\chi_{n}$ are Fourier sine series coefficients.

We know that

$$
\begin{gathered}
\Phi(x)= \begin{cases}\varphi(x), & x \in L, \\
0, & x \in L^{\prime},\end{cases} \\
\left(L^{\prime}=[0, l] \mid L\right), \\
\tau_{+}(x)= \begin{cases}\tau_{+}^{(0)}(x), & x \in L^{+}, \\
\tau(x), & x \in L^{\prime},\end{cases} \\
\tau_{-}(x)= \begin{cases}\tau_{-}^{(0)}, & x \in L^{-}, \\
0, & x \in L^{\prime} .\end{cases}
\end{gathered}
$$

Now from the first equation of (8) and (A.2)

$$
\begin{aligned}
& \Phi^{\prime}(x)= \begin{cases}\varphi^{\prime}(x) & (x \in L) \\
0 & \left(x \in L_{1}\right)\end{cases} \\
&=\frac{2}{l} \sum_{n=1}^{\infty} \frac{\pi n}{l} \Phi_{n} \cos \frac{\pi n x}{l} \\
&=\frac{2}{l} \sum_{n=1}^{\infty} \varphi_{n} \cos \frac{\pi n x}{l} \quad\left(\varphi_{n}=\frac{\pi n}{l} \Phi_{n}\right), \\
& \varphi_{n}=\int_{L} \varphi^{\prime}(x) \cos \frac{\pi n x}{l} d x \quad(n=1,2, \ldots) .
\end{aligned}
$$

Moreover we have

$$
\begin{aligned}
\chi_{n}= & \int_{0}^{l} \chi(x) \sin \frac{\pi n x}{l} d x \\
= & \int_{L}\left[\tau_{+}^{(0)}(x)-\tau_{-}^{(0)}(x)\right] \sin \frac{\pi n x}{l} d x \\
& (n=1,2, \ldots),
\end{aligned}
$$

$$
\Omega_{n}=-\frac{2 \pi n}{l}
$$

$$
\begin{gathered}
\cdot\left(G_{1} G_{2} \operatorname{sh}\left(\frac{\pi n h_{1}}{l}\right) \operatorname{sh}\left(\frac{\pi n h_{2}}{l}\right) \Phi_{n}\right) \\
\cdot\left(G_{1} \operatorname{sh}\left(\frac{\pi n h_{1}}{l}\right) \operatorname{ch}\left(\frac{\pi n h_{2}}{l}\right)\right. \\
\left.\left.+G_{2} \operatorname{sh}\left(\frac{\pi n h_{1}}{l}\right) \operatorname{ch}\left(\frac{\pi n h_{2}}{l}\right)\right)^{-1}\right) \\
+\left(\left(G_{1} \operatorname{sh}\left(\frac{\pi n h_{1}}{l}\right) \operatorname{ch}\left(\frac{\pi n h_{2}}{l}\right)\right.\right. \\
\left.-G_{2} \operatorname{ch}\left(\frac{\pi n h_{1}}{l}\right) \operatorname{sh}\left(\frac{\pi n h_{2}}{l}\right)\right) \\
+\left(G_{1} \operatorname{sh}\left(\frac{\pi n h_{1}}{l}\right) \operatorname{ch}\left(\frac{\pi n h_{2}}{l}\right)\right. \\
\left.\left.+G_{2} \operatorname{sh}\left(\frac{\pi n h_{2}}{l}\right) \operatorname{ch}\left(\frac{\pi n h_{1}}{l}\right)\right)^{-1}\right) \\
+\left(G_{1} \operatorname{sh}\left(\frac{\pi n h_{1}}{l}\right) \operatorname{ch}\left(\frac{\pi n h_{2}}{l}\right)\right. \\
+\left(\bar{\tau}_{1}(n) G_{2} \operatorname{sh}\left(\frac{\pi n h_{2}}{l}\right)+\bar{\tau}_{2}(n) G_{1} \operatorname{sh}\left(\frac{\pi n h_{1}}{l}\right)\right)
\end{gathered}
$$

in which

$$
\operatorname{sh}=\sinh , \quad \mathrm{ch}=\cosh .
$$

The two sides of (A.5) are multiplied by $(2 / l) \sin (\pi n x / l)$ and summed over index $n$; then based on (A.1) to (A.4), it yields

$$
\begin{aligned}
& \Omega(x) \\
& =-\frac{4}{l} G_{1} G_{2} \\
& \quad \cdot \sum_{n=1}^{\infty} \frac{\operatorname{sh}\left(\pi n h_{1} / 1\right) \operatorname{sh}\left(\pi n h_{2} / l\right) \varphi_{n} \sin (\pi n x / l)}{\operatorname{sh}\left(\pi n h_{1} / l\right) \operatorname{ch}\left(\pi n h_{2} / l\right)+G_{2} \operatorname{ch}\left(\pi n h_{1} / l\right) \operatorname{sh}\left(\pi n h_{2} / l\right)} \\
& +\frac{2}{l} \sum_{n=1}^{\infty} \frac{G_{1} \operatorname{sh}\left(\pi n h_{1} / l\right) \operatorname{ch}\left(\pi n h_{2} / l\right)-G_{2} \operatorname{ch}\left(\pi n h_{1} / l\right) \operatorname{sh}\left(\pi n h_{2} / l\right)}{G_{1} \operatorname{sh}\left(\pi n h_{1} / l\right) \operatorname{ch}\left(\pi n h_{2} / l\right)+G_{2} \operatorname{ch}\left(\pi n h_{1} / l\right) \operatorname{sh}\left(\pi n h_{2} / l\right)} \\
& \quad \cdot \chi_{n} \sin \frac{\pi n x}{l} \\
& +\frac{2}{l} \sum_{n=1}^{\infty} \frac{\bar{\tau}_{1}(n) G_{2} \operatorname{sh}\left(\pi n h_{2} / l\right)+\bar{\tau}_{2}(n) G_{1} \operatorname{sh}\left(\pi n h_{1} / l\right)}{G_{1} \operatorname{sh}\left(\pi n h_{1} / l\right) \operatorname{ch}\left(\pi n h_{2} / l\right)+G_{2} \operatorname{ch}\left(\pi n h_{1} / l\right) \operatorname{sh}\left(\pi n h_{2} / l\right)} \\
& \quad \cdot \sin \frac{\pi n x}{l} .
\end{aligned}
$$


Now introducing the below dimensionless parameters

$$
\begin{gathered}
h_{+}=\frac{\pi h_{1}}{l}, \quad h_{-}=\frac{\pi h_{2}}{l} ; \quad \xi=\frac{\pi x}{l}, \quad \eta=\frac{\pi s}{l} ; \\
(0<\xi, \eta<\pi), \\
\mu=\frac{G_{1}}{G_{2}}, \quad \Omega_{0}(\xi)=\frac{\Omega(l \xi / \pi)}{G_{1}}
\end{gathered}
$$

and substituting into the functions $\bar{\tau}_{+}(n), \bar{\tau}_{1}(n)$ and also $\bar{\tau}_{-}(n), \bar{\tau}_{2}(n)$, regarding (A.2), we have

(1)

$$
\begin{aligned}
\bar{\tau}_{+}(n) & =\int_{0}^{l} \tau_{+}(s) \sin \frac{\pi n s}{l} d s \\
& =\frac{l}{\pi} \int_{0}^{\pi} \tau_{+}\left(\frac{l \eta}{\pi}\right) \sin n \eta d \eta \\
& =\frac{l G_{1}}{\pi} \int_{0}^{\pi} \bar{\tau}_{+}(\eta) \sin n \eta d \eta, \\
& \bar{\tau}_{+}(\eta)=\frac{\tau_{+}(l \eta / \pi)}{G_{1}}
\end{aligned}
$$

also supposing

$$
\alpha_{k}=\frac{\pi a_{k}}{l}, \quad \beta_{k}=\frac{\pi b_{k}}{l} \quad(k=\overline{1, N}) .
$$

In this case the system $L$ converts to the system $L_{0}=$ $\cup_{k=1}^{N}\left(\alpha_{k}, \beta_{k}\right)$ and $L_{1}^{\prime}=[0, \pi] \mid L_{0}$.

Regarding (A.2) we can write

$$
\begin{array}{r}
\tilde{\tau}_{+}(\xi)=\frac{\tau_{+}^{0}(l \xi / \pi)}{G_{1}}, \\
\tilde{\tau}(\xi)=\frac{\tau(l \xi / \pi)}{G_{1}}, \\
\tilde{\tau}_{-}(\xi)=\frac{\tau_{-}^{0}(l \xi / \pi)}{G_{1}} \\
(0<\xi<\pi)
\end{array}
$$

also supposing

(2)

$$
\bar{\tau}_{k}(\xi)=\frac{\tau_{k}(l \xi / \pi)}{G_{1}} \quad(k=1,2) .
$$

In this manner we have

$$
\begin{aligned}
& \bar{\tau}_{1}(n)= \int_{0}^{l} \tau_{1}(s) \sin \frac{\pi n s}{l} d s \\
&= \frac{l}{\pi} \int_{0}^{\pi} \tau_{1}\left(\frac{l \eta}{\pi}\right) \sin n \eta d \eta \\
&= \frac{l G_{1}}{\pi} \int_{0}^{\pi} \bar{\tau}_{1}(\eta) \sin n \eta d \eta \\
&(n=1,2, \ldots)
\end{aligned}
$$

(3)

$$
\begin{aligned}
\bar{\tau}_{-}(n) & =\int_{0}^{l} \tau_{-}(s) \sin \frac{\pi n s}{l} d s \\
& =\frac{l}{\pi} \int_{0}^{\pi} \tau_{-}\left(\frac{l \eta}{\pi}\right) \sin n \eta d \eta \\
& =\frac{l G_{1}}{\pi} \int_{0}^{\pi} \bar{\tau}_{-}(\eta) \sin n \eta d \eta, \\
& \bar{\tau}_{-}(\eta)=\frac{\tau_{-}(\operatorname{l\eta } / \pi)}{G_{1}}
\end{aligned}
$$

(4)

$$
\begin{aligned}
& \bar{\tau}_{2}(n)= \int_{0}^{l} \tau_{2}(s) \sin \frac{\pi n s}{l} d s \\
&= \frac{l}{\pi} \int_{0}^{\pi} \tau_{2}\left(\frac{l \eta}{\pi}\right) \sin n \eta d \eta \\
&= \frac{l G_{1}}{\pi} \int_{0}^{\pi} \bar{\tau}_{2}(\eta) \sin n \eta d \eta \\
&(n=1,2, \ldots)
\end{aligned}
$$

(5)

$$
\begin{aligned}
\varphi_{n} & =\int_{L} \varphi^{\prime}(x) \cos \frac{\pi n x}{l} d x \\
& =\frac{l}{\pi} \int_{L_{0}} \varphi^{\prime}\left(\frac{l \eta}{\pi}\right) \cos n \eta d \eta \\
& =\frac{l}{\pi} \int_{L_{0}} \varphi_{0}(\eta) \cos n \eta d \eta \\
& \left(\varphi_{0}(\xi)=\varphi^{\prime}\left(\frac{l \xi}{\pi}\right)\right)
\end{aligned}
$$

(6)

$$
\begin{aligned}
\chi_{n} & =\int_{L}\left[\tau_{+}^{(0)}(s)-\tau_{-}^{(0)}(s)\right] \sin \frac{\pi n s}{l} d s \\
& =\frac{l}{\pi} \int_{L_{0}}\left[\tau_{+}^{(0)}\left(\frac{l \eta}{\pi}\right)-\tau_{-}^{(0)}\left(\frac{l \eta}{\pi}\right)\right] \sin n \eta d \eta \\
& =\frac{l G_{1}}{\pi} \int_{L_{0}}\left[\tilde{\tau}_{+}(\eta)-\tilde{\tau}_{-}(\eta)\right] \sin n \eta d \eta
\end{aligned}
$$$$
(n=1,2, \ldots) \text {. }
$$

Using all the above equations and parameters into (A.7), it yields

$$
\begin{aligned}
& \Omega_{0}(\xi) \\
& =-\frac{4}{\pi} \sum_{n=1}^{\infty} \frac{\operatorname{sh}\left(n h_{+}\right) \operatorname{sh}\left(n h_{-}\right) \sin n \xi}{\operatorname{sh}\left(n h_{+}\right) \operatorname{ch}\left(n h_{-}\right)+\operatorname{ch}\left(n h_{+}\right) \operatorname{sh}\left(n h_{-}\right)} \\
& \quad \cdot \int_{L_{0}} \varphi_{0}(\eta) \cos n \eta d \eta
\end{aligned}
$$




$$
\begin{aligned}
& +\frac{2}{\pi} \sum_{n=1}^{\infty} \frac{\mu \operatorname{sh}\left(n h_{+}\right) \operatorname{ch}\left(n h_{-}\right)-\operatorname{ch}\left(n h_{+}\right) \operatorname{sh}\left(n h_{-}\right)}{\mu \operatorname{sh}\left(n h_{+}\right) \operatorname{ch}\left(n h_{-}\right)+\operatorname{ch}\left(n h_{+}\right) \operatorname{sh}\left(n h_{-}\right)} \\
& \cdot \int_{L_{0}}\left[\tilde{\tau}_{+}(\eta)-\widetilde{\tau}_{-}(\eta)\right] \sin n \eta d \eta \\
& +\frac{2}{\pi} \sum_{n=1}^{\infty} \frac{\operatorname{sh}\left(n h_{-}\right) \sin n \xi}{\mu \operatorname{sh}\left(n h_{+}\right) \operatorname{ch}\left(n h_{-}\right)+\operatorname{ch}\left(n h_{+}\right) \operatorname{sh}\left(n h_{-}\right)} \\
& \cdot \int_{0}^{\pi} \bar{\tau}_{1}(\eta) \sin n \eta d \eta \\
& +\frac{2}{\pi} \sum_{n=1}^{\infty} \frac{\mu \operatorname{sh}\left(n h_{+}\right) \sin n \xi}{\left.\mu h_{+}\right) \operatorname{ch}\left(n h_{-}\right)+\operatorname{ch}\left(n h_{+}\right) \operatorname{sh}\left(n h_{-}\right)} \\
& \cdot \int_{0}^{\pi} \bar{\tau}_{2}(\eta) \sin n \eta d \eta .
\end{aligned}
$$

Now we change the integral sign with the summation sign; the above equation converts to

$$
\begin{aligned}
& \Omega_{0}(\xi) \\
& =-\frac{4}{\pi} \int_{L_{0}} \sum_{n=1}^{\infty} \frac{\operatorname{sh}\left(n h_{+}\right) \operatorname{sh}\left(n h_{-}\right) \sin n \xi \cos n \eta}{\mu \operatorname{sh}\left(n h_{+}\right) \operatorname{ch}\left(n h_{-}\right)+\operatorname{ch}\left(n h_{+}\right) \operatorname{sh}\left(n h_{-}\right)} \\
& +\frac{2}{\pi} \int_{L_{0}} \sum_{n=1}^{\infty} \frac{\mu \operatorname{sh}\left(n h_{+}\right) \operatorname{ch}\left(n h_{-}\right)-\operatorname{ch}\left(n h_{+}\right) \operatorname{sh}\left(n h_{-}\right)}{\mu \operatorname{sh}\left(n h_{+}\right) \operatorname{ch}\left(n h_{-}\right)+\operatorname{ch}\left(n h_{+}\right) \operatorname{sh}\left(n h_{-}\right)} \\
& +\frac{2}{\pi} \int_{0}^{\pi} \sum_{n=1}^{\infty} \frac{\sin n \xi \sin n \eta\left[\widetilde{\tau}_{+}(\eta)-\widetilde{\tau}_{-}(\eta)\right] d \eta}{\mu \operatorname{sh}\left(n h_{+}\right) \operatorname{ch}\left(n h_{-}\right)+\operatorname{ch}\left(n h_{+}\right) \operatorname{sh}\left(n h_{-}\right)} \\
& +\frac{2}{\pi} \int_{0}^{\pi} \sum_{n=1}^{\infty} \frac{\mu \sin \left(n h_{+}\right) \sin n \xi \sin n \eta}{\mu \operatorname{sh}\left(n h_{+}\right) \operatorname{ch}\left(n h_{-}\right)+\operatorname{ch}\left(n h_{+}\right) \operatorname{sh}\left(n h_{-}\right)} \\
& \cdot \bar{\tau}_{2}(\eta) d \eta .
\end{aligned}
$$

Based on the mathematics

$$
\operatorname{sh}\left(n h_{ \pm}\right) \approx e^{n h_{ \pm}}, \quad \operatorname{ch}\left(n h_{ \pm}\right) \approx e^{n h_{ \pm}}
$$$$
\text { in which } n \longrightarrow \infty \text {. }
$$

Now we change the two first quantities of (A.19) accordingly
(1)

$$
\begin{aligned}
& \sum_{n=1}^{\infty} \frac{\operatorname{sh}\left(n h_{+}\right) \operatorname{sh}\left(n h_{-}\right) \sin n \xi \cos n \eta}{\mu \operatorname{sh}\left(n h_{+}\right) \operatorname{ch}\left(n h_{-}\right)+\operatorname{ch}\left(n h_{+}\right) \operatorname{sh}\left(n h_{-}\right)} \\
& =\sum_{n=1}^{\infty}\left[\frac{\operatorname{sh}\left(n h_{+}\right) \operatorname{sh}\left(n h_{-}\right)}{\mu \operatorname{sh}\left(n h_{+}\right) \operatorname{ch}\left(n h_{-}\right)+\operatorname{ch}\left(n h_{+}\right) \operatorname{sh}\left(n h_{-}\right)}-\frac{1}{\mu+1}\right] \\
& \quad \cdot \sin n \xi \cos n \eta \\
& +\frac{1}{\mu+1} \sum_{n=1}^{\infty} \sin n \xi \cos n \eta \\
& =\frac{1}{2(\mu+1)}\left[\sum_{n=1}^{\infty} \sin n(\xi+\eta)+\sum_{n=1}^{\infty} \sin n(\xi-\eta)\right] \\
& +\sum_{n=1}^{\infty}\left[\left(\mu \operatorname{sh}\left(n h_{+}\right)\left(\operatorname{sh}\left(n h_{-}\right)-\operatorname{ch}\left(n h_{-}\right)\right)\right.\right. \\
& \left.\quad+\operatorname{sh}\left(n h_{-}\right)\left(\operatorname{sh}\left(n h_{+}\right)-\operatorname{ch}\left(n h_{+}\right)\right)\right) \\
& \quad\left(( \mu + 1 ) \left[\mu \operatorname{sh}\left(n h_{+}\right) \operatorname{ch}\left(n h_{-}\right)\right.\right. \\
& \left.\left.\left.+\operatorname{ch}\left(n h_{+}\right) \operatorname{sh}\left(n h_{-}\right)\right]\right)^{-1}\right]
\end{aligned}
$$

$\cdot \sin n \xi \cos n \eta$

$$
\begin{aligned}
&= \frac{1}{\mu+1} \sum_{n=1}^{\infty} \frac{-\mu \operatorname{sh}\left(n h_{+}\right) e^{-n h_{-}}-\operatorname{sh}\left(n h_{-}\right) e^{-n h_{+}}}{\left[\mu \operatorname{sh}\left(n h_{+}\right) \operatorname{ch}\left(n h_{-}\right)+\operatorname{ch}\left(n h_{+}\right) \operatorname{sh}\left(n h_{-}\right)\right]} \\
& \cdot \sin n \xi \cos n \eta \\
&=\frac{1}{2(\mu+1)}\left[\sum_{n=1}^{\infty} \sin n(\xi+\eta)+\sum_{n=1}^{\infty} \sin n(\xi-\eta)\right] \\
&=\frac{1}{2(\mu+1)}\left[\operatorname{ctg} \frac{\xi-\eta}{2}+\operatorname{ctg} \frac{\xi+\eta}{2}\right] \\
&-\frac{1}{\mu+1} \sum_{n=1}^{\infty} \frac{\mu \operatorname{sh}\left(n h_{+}\right) e^{-n h_{-}}+\operatorname{sh}\left(n h_{-}\right) e^{-n h_{+}}}{\left[\mu \operatorname{sh}\left(n h_{+}\right) \operatorname{ch}\left(n h_{-}\right)+\operatorname{ch}\left(n h_{+}\right) \operatorname{sh}\left(n h_{-}\right)\right]} \\
& \cdot \sin n \xi \cos n \eta
\end{aligned}
$$

and then using the equality

$$
\begin{aligned}
\sum_{n=1}^{\infty} \frac{\cos n \xi}{n} & =\ln \frac{1}{2|\sin (\xi / 2)|} \text { and so } \\
\frac{1}{2} \operatorname{ctg} \frac{\xi}{2}= & \sum_{n=1}^{\infty} \sin n \xi \\
& (0<\xi<2 \pi)
\end{aligned}
$$

(2)

$\sum_{n=1}^{\infty} \frac{\mu \operatorname{sh}\left(n h_{+}\right) \operatorname{ch}\left(n h_{-}\right)-\operatorname{ch}\left(n h_{+}\right) \operatorname{sh}\left(n h_{-}\right)}{\mu \operatorname{sh}\left(n h_{+}\right) \operatorname{ch}\left(n h_{-}\right)+\operatorname{ch}\left(n h_{+}\right) \operatorname{sh}\left(n h_{-}\right)} \sin n \xi \sin n \eta$

$$
=\sum_{n=1}^{\infty}\left[\frac{\operatorname{sh}\left(n h_{+}\right) \operatorname{sh}\left(n h_{-}\right)}{\mu \operatorname{sh}\left(n h_{+}\right) \operatorname{ch}\left(n h_{-}\right)+\operatorname{ch}\left(n h_{+}\right) \operatorname{sh}\left(n h_{-}\right)}-\frac{\mu-1}{\mu+1}\right]
$$

$\cdot \sin n \xi \sin n \eta$ 


$$
\begin{aligned}
& +\frac{\mu-1}{2(\mu+1)}\left[\sum_{n=1}^{\infty} \cos n(\xi-\eta)-\sum_{n=1}^{\infty} \cos n(\xi+\eta)\right] \\
& =\frac{2 \mu}{\mu+1} \sum_{n=1}^{\infty} \frac{\operatorname{sh} n\left(h_{+}-h_{-}\right) \sin n \xi \sin n \eta}{\left(n h_{+}\right) \operatorname{ch}\left(n h_{-}\right)+\operatorname{ch}\left(n h_{+}\right) \operatorname{sh}\left(n h_{-}\right)} \\
& \quad+\frac{\pi(\mu-1)}{2(\mu+1)}[\delta(\xi-\eta)-\delta(\xi+\eta)] .
\end{aligned}
$$

In the same manner

$$
\begin{aligned}
& \sum_{n=1}^{\infty} \frac{\sin n \xi}{n}=\frac{\pi-|x|}{2} \operatorname{sign} x \quad(-2 \pi<x<2 \pi) \\
& \text { from that } \sum_{n=1}^{\infty} \cos n x=\pi \delta(x)-\frac{1}{2} .
\end{aligned}
$$

Using the above conversations, (A.19) can be written as follows:

$$
\begin{aligned}
\Omega_{0}(\xi)= & -\frac{1}{\pi(\mu+1)} \int_{L_{0}}\left[\operatorname{ctg} \frac{\xi-\eta}{2}+\operatorname{ctg} \frac{\xi+\eta}{2}\right] \varphi_{0}(\eta) d \eta \\
& +\frac{4}{\pi(\mu+1)} \\
& \cdot \int_{L_{0}}\left(\sum_{n=1}^{\infty} \frac{\mu \operatorname{sh}\left(n h_{+}\right) e^{-n h_{-}}+\operatorname{sh}\left(n h_{-}\right) e^{-n h_{+}}}{\left[\mu \operatorname{sh}\left(n h_{+}\right) \operatorname{ch}\left(n h_{-}\right)+\operatorname{ch}\left(n h_{+}\right) \operatorname{sh}\left(n h_{-}\right)\right]}\right. \\
& +\frac{\mu-1}{\mu+1} \int_{L_{0}}[\delta(\xi-\eta)-\delta(\xi+\eta)]\left[\tilde{\tau}_{+}(\eta)-\tilde{\tau}_{-}(\eta)\right] d \eta \\
& +\frac{4 \mu}{\pi(\mu+1)} \sin n \eta \varphi_{0}(\eta) d \eta \\
& \cdot \int_{L_{0}}\left(\sum_{n=1}^{\infty} \frac{\operatorname{sh} n\left(h_{+}-h_{-}\right) \sin n \xi \sin n \eta}{\left(n h_{+}\right) \operatorname{ch}\left(n h_{-}\right)+\operatorname{ch}\left(n h_{+}\right) \operatorname{sh}\left(n h_{-}\right)}\right) \\
& \cdot\left[\tilde{\tau}_{+}(\eta)-\tilde{\tau}_{-}(\eta)\right] d \eta \\
& +\frac{2}{\pi} \int_{0}^{\pi}\left(\sum_{n=1}^{\infty} \frac{\operatorname{sh} n h_{-} \sin n \xi \sin n \eta}{\left(n h_{+}\right) \operatorname{ch}\left(n h_{-}\right)+\operatorname{ch}\left(n h_{+}\right) \operatorname{sh}\left(n h_{-}\right)}\right) \\
& \cdot \bar{\tau}_{1}(\eta) d \eta \\
& +\frac{2}{\pi} \int_{0}^{\pi}\left(\sum_{n=1}^{\infty} \frac{\mu \operatorname{sh} n h_{+} \sin n \xi \sin n \eta}{2\left(\eta h_{+}\right) \operatorname{ch}\left(n h_{-}\right)+\operatorname{ch}\left(n h_{+}\right) \operatorname{sh}\left(n h_{-}\right)}\right) \\
& (0<\pi)
\end{aligned}
$$

considering the below kernels

$$
\begin{aligned}
& K_{*}(\xi)=\sum_{n=1}^{\infty} \frac{\mu \operatorname{sh}\left(n h_{+}\right) e^{-n h_{-}}+\operatorname{sh}\left(n h_{-}\right) e^{-n h_{+}}}{\left[\mu \operatorname{sh}\left(n h_{+}\right) \operatorname{ch}\left(n h_{-}\right)+\operatorname{ch}\left(n h_{+}\right) \operatorname{sh}\left(n h_{-}\right)\right]} \sin n \xi \\
& L_{*}(\xi)=\sum_{n=1}^{\infty} \frac{\operatorname{sh} n\left(h_{+}-h_{-}\right) \cos n \xi}{\mu \operatorname{sh}\left(n h_{+}\right) \operatorname{ch}\left(n h_{-}\right)+\operatorname{ch}\left(n h_{+}\right) \operatorname{sh}\left(n h_{-}\right)}
\end{aligned}
$$

$$
\begin{aligned}
L_{1}^{*}(\xi) & =\sum_{n=1}^{\infty} \frac{\operatorname{sh} n h_{-} \cos n \xi}{\mu \operatorname{sh}\left(n h_{+}\right) \operatorname{ch}\left(n h_{-}\right)+\operatorname{ch}\left(n h_{+}\right) \operatorname{sh}\left(n h_{-}\right)} \\
L_{2}^{*}(\xi) & =\mu \sum_{n=1}^{\infty} \frac{\operatorname{sh} n h_{+} \cos n \xi}{\mu \operatorname{sh}\left(n h_{+}\right) \operatorname{ch}\left(n h_{-}\right)+\operatorname{ch}\left(n h_{+}\right) \operatorname{sh}\left(n h_{-}\right)}
\end{aligned}
$$

For simplicity, (A.25) can be written as

$$
\begin{aligned}
\Omega_{0}(\xi)= & -\frac{1}{\pi(\mu+1)} \int_{L_{0}}\left[\operatorname{ctg} \frac{\xi-\eta}{2}+\operatorname{ctg} \frac{\xi+\eta}{2}\right] \varphi_{0}(\eta) d \eta \\
& +\frac{2}{\pi(\mu+1)} \int_{L_{0}}\left(K_{*}(\xi-\eta)+K_{*}(\xi+\eta)\right) \\
& \cdot \varphi_{0}(\eta) d \eta \\
& +\frac{\mu-1}{\mu+1} \int_{L_{0}}[\delta(\xi-\eta)-\delta(\xi+\eta)] \\
& +\frac{2 \mu}{\pi(\mu+1)} \int_{L_{0}}\left(L_{*}(|\xi-\eta|)-L_{*}(\xi+\eta)\right) \\
& +\frac{1}{\pi} \int_{0}^{\pi}\left(L_{1}^{*}(|\xi-\eta|)-L_{1}^{*}(\xi+\eta)\right) \bar{\tau}_{1}(\eta) d \eta \\
& +\frac{1}{\pi} \int_{0}^{\pi}\left(L_{2}^{*}(|\xi-\eta|)-\tilde{\tau}_{2}^{*}(\xi+\eta)\right) \bar{\tau}_{2}(\eta) d \eta
\end{aligned}
$$

and it is clear that

$$
\begin{aligned}
\int_{L_{0}} & {[\delta(\xi-\eta)-\delta(\xi+\eta)]\left[\tilde{\tau}_{+}(\eta)-\tilde{\tau}_{-}(\eta)\right] d \eta } \\
= & \int_{L_{0}} \delta(\xi-\eta)\left[\tilde{\tau}_{+}(\eta)-\tilde{\tau}_{-}(\eta)\right] d \eta \\
& -\int_{L_{0}} \delta(\xi+\eta)\left[\tilde{\tau}_{+}(\eta)-\tilde{\tau}_{-}(\eta)\right] d \eta .
\end{aligned}
$$

In the second integral, by changing $\eta \rightarrow-\eta$, because of odd function, we have

$$
\begin{aligned}
& \tilde{\tau}_{+}(-\eta)-\tilde{\tau}_{-}(-\eta)=-\left[\tilde{\tau}_{+}(\eta)-\tilde{\tau}_{-}(\eta)\right], \\
& L_{0}=\sum_{k=1}^{N}\left(\alpha_{k}, \beta_{k}\right), \\
& \tilde{L}_{0}=\sum_{k=1}^{N}\left(-\beta_{k},-\alpha_{k}\right) .
\end{aligned}
$$


From that it can be concluded that

$$
\begin{aligned}
\int_{L_{0}} & {[\delta(\xi-\eta)-\delta(\xi+\eta)]\left[\tilde{\tau}_{+}(\eta)-\tilde{\tau}_{-}(\eta)\right] d \eta } \\
= & \int_{L_{0}} \delta(\xi-\eta)\left[\tilde{\tau}_{+}(\eta)-\tilde{\tau}_{-}(\eta)\right] d \eta \\
& -\int_{L_{0}} \delta(\xi+\eta)\left[\widetilde{\tau}_{+}(\eta)-\tilde{\tau}_{-}(\eta)\right] d \eta \\
= & \int_{L_{0}+\tilde{L}_{0}} \delta(\xi-\eta)\left[\tilde{\tau}_{+}(\eta)-\tilde{\tau}_{-}(\eta)\right] d \eta \\
= & \int_{L_{0}+\tilde{L}_{0}} \delta(\eta-\xi)\left[\widetilde{\tau}_{+}(\eta)-\widetilde{\tau}_{-}(\eta)\right] d \eta \\
= & \widetilde{\tau}_{+}(\xi)-\tilde{\tau}_{-}(\xi) .
\end{aligned}
$$

Therefore (A.27) changes to the following form:

$$
\begin{aligned}
\Omega_{0}(\xi)= & -\frac{1}{\pi(\mu+1)} \int_{L_{0}}\left[\operatorname{ctg} \frac{\xi-\eta}{2}+\operatorname{ctg} \frac{\xi+\eta}{2}\right] \varphi_{0}(\eta) d \eta \\
& +\frac{2}{\pi(\mu+1)} \int_{L_{0}}\left(K_{*}(\xi-\eta)+K_{*}(\xi+\eta)\right) \\
& \cdot \varphi_{0}(\eta) d \eta \\
& +\frac{\mu-1}{\mu+1}\left[\tilde{\tau}_{+}(\xi)-\tilde{\tau}_{-}(\xi)\right]+\frac{2 \mu}{\pi(\mu+1)} \\
& \cdot \int_{L_{0}}\left(L_{*}(|\xi-\eta|)-L_{*}(\xi+\eta)\right) \\
& +\frac{1}{\pi} \int_{0}^{\pi}\left(L_{1}^{*}(|\xi-\eta|)-L_{1}^{*}(\xi+\eta)\right) \bar{\tau}_{1}(\eta) d \eta \\
& +\frac{1}{\pi} \int_{0}^{\pi}\left(L_{2}^{*}(|\xi-\eta|)-L_{2}^{*}(\xi+\eta)\right) \bar{\tau}_{2}(\eta) d \eta .
\end{aligned}
$$

Now in the cracks system $L$ we have

$$
\begin{array}{r}
\Omega(x)=\frac{\tau_{+}(x)+\tau_{-}(x)}{2}=\frac{\tau_{+}^{(0)}(x)+\tau_{-}^{(0)}(x)}{2} \\
(x \in L) \quad\left(\xi=\frac{\pi x}{l}\right)
\end{array}
$$

and in the system $L_{0}$

$$
\Omega_{0}(\xi)=\frac{\tilde{\tau}_{+}(\xi)+\tilde{\tau}_{-}(\xi)}{2} \quad\left(\xi \in L_{0}\right) .
$$

In continuation, (A.31) reduces to the following problem in system $L_{0}$ :

$$
\begin{aligned}
\Omega_{0}(\xi)= & -\frac{1}{\pi(\mu+1)} \int_{L_{0}}\left[\operatorname{ctg} \frac{\xi-\eta}{2}+\operatorname{ctg} \frac{\xi+\eta}{2}\right] \varphi_{0}(\eta) d \eta \\
& +\frac{2}{\pi(\mu+1)} \\
& \cdot \int_{L_{0}}\left(K_{*}(\xi-\eta)+K_{*}(\xi+\eta)\right) \varphi_{0}(\eta) d \eta=f(\xi)
\end{aligned}
$$

in which we have

$$
\begin{aligned}
\tilde{f}(\xi)= & \frac{\tilde{\tau}_{+}(\xi)+\tilde{\tau}_{-}(\xi)}{2}-\frac{\mu-1}{\mu+1}\left[\tilde{\tau}_{+}(\xi)-\tilde{\tau}_{-}(\xi)\right] \\
& -\frac{2 \mu}{\pi(\mu+1)} \\
& \cdot \int_{L_{0}}\left(L_{*}(|\xi-\eta|)-L_{*}(\xi+\eta)\right)\left[\tilde{\tau}_{+}(\eta)-\tilde{\tau}_{-}(\eta)\right] d \eta \\
& +\frac{1}{\pi} \int_{0}^{\pi}\left(L_{1}^{*}(|\xi-\eta|)-L_{1}^{*}(\xi+\eta)\right) \bar{\tau}_{1}(\eta) d \eta \\
& -\frac{1}{\pi} \int_{0}^{\pi}\left(L_{2}^{*}(|\xi-\eta|)-L_{2}^{*}(\xi+\eta)\right) \bar{\tau}_{2}(\eta) d \eta .
\end{aligned}
$$

\section{Abbreviations}

$a_{k}: \quad$ Beginning tip of crack

$b_{k}: \quad$ End tip of crack

COD: Crack opening displacement

$D_{1}$ : $\quad$ Top rectangular plate

$\mathrm{D}_{2}$ : $\quad$ Bottom rectangular plate

$G$ : $\quad$ Shear modulus

$h_{0}$ : Height parameter

$h_{1}, h_{2}$ : Heights of top and bottom rectangles

$k=G_{0} / G$ : Shear moduli ratio

$K_{\text {III }}^{(0)}: \quad$ Dimensionless antiplane SIF

$l$ : $\quad$ Length of plate

L: $\quad$ Total length of cracks

$L^{\prime}$ : $\quad$ Length of noncracked region on interface line

$L^{+}, L^{-}: \quad$ Upper and lower boundaries of cracks

$L_{k}$ : $\quad$ Length of one crack

$M: \quad$ Arbitrary natural number

$N: \quad$ Number of cracks

$P$ : $\quad$ Antiplane point load at midpoint of plate edges

$r, \rho: \quad$ Variable for changing of integration domain

SIE: $\quad$ Singular integral equation

sh: $\quad$ sinh

ch: $\quad$ cosh

SIF: $\quad$ Stress intensity factor 


$\begin{array}{ll}T(x): & \begin{array}{l}\text { Antiplane distributed shear traction on } \\ \text { edges of plate }\end{array} \\ T_{n}(u): & \text { Chebyshev polynomial of the first kind } \\ U_{n-1}(u): & \text { Chebyshev polynomial of the first kind } \\ \alpha, \beta: & \text { Parameters for the beginning and end } \\ & \text { tips of crack } \\ \tau_{+}(x): & \text { Antiplane shear stress of upper rectangle } \\ \tau_{-}(x): & \text { Antiplane shear stress of lower rectangle } \\ \tau_{ \pm}^{(0)}: & \text { Varying internal loading on crack edges } \\ \tau_{y z}^{(1)}: & \text { Antiplane shear stress of upper rectangle } \\ \tau_{y z}^{(2)}: & \text { Antiplane shear stress of lower rectangle } \\ \tau_{1}(x): & \text { Antiplane shear loading at the top edge } \\ \tau_{2}(x): & \text { of body } \\ \mu=G / G_{2}: & \text { Antiplane shear loading at the bottom } \\ \lambda=k l=\left(G_{0} / G\right) l: & \text { Shear moduli ratio parameter } \\ \xi, \eta: & \text { Length parameters for shear stress } \\ \delta(x): & \text { calculations } \\ \omega(x, y): & \text { Dirac delta function } \\ \Lambda_{0}: & \text { Displacement function along } z \text {-axis } \\ \Phi(x), \varphi(x): & \text { Domain of crack length parameters } \\ \Omega(x), X(x): & \text { Displacement functions } \\ & \text { Stress functions. }\end{array}$

\section{Conflict of Interests}

The authors declare that there is no conflict of interests regarding the publication of this paper.

\section{References}

[1] L. P. Pook, F. Berto, A. Campagnolo, and P. Lazzarin, "Couple fracture mode of a cracked disc under antiplane loading," Engineering Fracture Mechanics, vol. 128, pp. 22-36, 2014.

[2] L. P. Pook, "A 50-year retrospective review of three-dimensional effects at cracks and sharp notches," Fatigue and Fracture of Engineering Materials and Structures, vol. 36, no. 8, pp. 699-723, 2013.

[3] A. Kotousov, P. Lazzarin, F. Berto, and L. P. Pook, "Threedimensional stress states at crack tip induced by shear and antiplane loading," Engineering Fracture Mechanics, vol. 108, pp. 6574, 2013.

[4] L. P. Pook, A. Campagnolo, F. Berto, and P. Lazzarin, "Coupled fracture mode of a cracked plate under anti-plane loading," Engineering Fracture Mechanics, vol. 134, pp. 391-403, 2015.

[5] L. Harold, Fracture: An Advanced Treatise Mathematical Fundamentals, vol. 1, Academic Press, New York, NY, USA, 1968.

[6] G. P. Cherepanov, Fracture Mechanics, Nauka, Moscow, Russia, 1974.

[7] V. V. Panasjuk, M. P. Savruk, and A. P. Datsyshyn, The Stress Distribution in The Neighborhood of Cracks of Plates and Shells, Naukova Dumka, Kiev, Ukraine, 1976.

[8] Y. Murakami, Stress Intensity Factors Handbook, vol. 1, Pergamon Press, New York, NY, USA, 1987.

[9] Y. Murakami, Stress Intensity Factors Handbook 2, Pergamon Press, New York, NY, USA, 1987.
[10] M. P. Savruk, “The stress intensity factors in cracked bodies," in Fracture Mechanics and Strength of Materials Handbook, vol. 2, p. 619, 1988.

[11] M. Zappalorto and P. Lazzarin, "Three-dimensional elastic stress fields ahead of notches in thick plates under various loading conditions," Engineering Fracture Mechanics, vol. 108, pp. 75-88, 2013.

[12] A. Kotousov, F. Berto, P. Lazzarin, and F. Pegorin, "Three dimensional finite element mixed fracture mode under antiplane loading of a crack," Theoretical and Applied Fracture Mechanics, vol. 62, no. 1, pp. 26-33, 2012.

[13] A. Campagnolo, F. Berto, and P. Lazzarin, "The effects of different boundary conditions on three-dimensional cracked discs under anti-plane loading," European Journal of Mechanics A: Solids, vol. 50, pp. 76-86, 2015.

[14] A. Boulenouar, N. Benseddiq, and M. Mazari, "Strain energy density prediction of crack propagation for 2D linear elastic materials," Theoretical and Applied Fracture Mechanics, vol. 6768, pp. 29-37, 2013.

[15] J. Fu, K. Hu, Z. Chen, L. Chen, and L. Qian, "A moving crack propagating in a functionally graded magnetoelectroelastic strip under different crack face conditions," Theoretical and Applied Fracture Mechanics, vol. 66, pp. 16-25, 2013.

[16] P. C. Gope, N. Bisht, and V. K. Singh, "Influence of crack offset distance on interaction of multiple collinear and offset edge cracks in a rectangular plate," Theoretical and Applied Fracture Mechanics, vol. 70, pp. 19-29, 2014.

[17] D. Misseroni, F. Dal Corso, S. Shahzad, and D. Bigoni, "Stress concentration near stiff inclusions: validation of rigid inclusion model and boundary layers by means of photo elasticity," Engineering Fracture Mechanics, vol. 121-122, pp. 87-97, 2014.

[18] G. Noselli, F. Dal Corso, and D. Bigoni, "The stress intensity near a stiffener disclosed by photoelasticity," International Journal of Fracture, vol. 166, no. 1-2, pp. 91-103, 2010.

[19] R. Ghajar and A. Shaghaghi Moghaddam, "Numerical investigation of the mode III stress intensity factors in FGMs considering the effect of graded Poisson's ratio," Engineering Fracture Mechanics, vol. 78, no. 7, pp. 1478-1486, 2011

[20] A. Hernández-Pérez, F. Avilés, and L. A. Carlsson, "Evaluation of the plate twist test to characterize mode III fracture of sandwich panels with a face/core interface crack," Engineering Fracture Mechanics, vol. 104, pp. 41-55, 2013.

[21] H. Okada, H. Kawai, and K. Araki, "A virtual crack closureintegral method (VCCM) to compute the energy release rates and stress intensity factors based on quadratic tetrahedral finite elements," Engineering Fracture Mechanics, vol. 75, no. 15, pp. 4466-4485, 2008.

[22] D. Chang and A. Kotousov, "A strip yield model for two collinear cracks in plates of arbitrary thickness," International Journal of Fracture, vol. 176, no. 1, pp. 39-47, 2012.

[23] D. Chang and A. Kotousov, "A strip yield model for two collinear cracks," Engineering Fracture Mechanics, vol. 90, pp. 121-128, 2012.

[24] V. A. Kryven, "Antiplane deformation of an elasto plastic body weakened by a double periodic system of notches," FizykoKhimichna Mekanika Materialiv, vol. 1, pp. 31-33, 1979.

[25] V. A. Kryvn, G. T. Sulym, and M. I. Yavorska, "Plastic interfacial slip of periodic systems of rigid thin inclusions undergoing longitudinal shear," Theoretical and Applied Fracture Mechanics, vol. 44, pp. 837-848, 2006.

[26] I. N. Sneddon, Fourier Transforms, Courier, New York, NY, USA, 1951. 
[27] P. S. Theocharis and N. I. Iokimidis, "Numerical integration methods for the solution of singular integral equations," Quarterly of Applied Mathematics, vol. 1, no. 35, pp. 173-185, 1977.

[28] F. Erdogan, G. D. Gupta, and T. S. Cook, "The numerical solutions of singular integral equations," in Methods of Analysis and Solution of Crack Problems Mechanics of Fracture, vol. 1, pp. 368-425, 1973.

[29] D. I. Bardzokas, S. H. Gevorgyan, and S. M. Mkhitaryan, "About a stress deformation condition of a piecewise-uniform wedge with a system of collinear cracks at an antiplane deformation," Mathematical Problems in Engineering, vol. 2005, no. 2, pp. 245268, 2005.

[30] S. M. Mkhitaryan and A. G. Pahlaviani, “The stress distribution field of piecewise homogeneous elastic rectangular plate with a system of collinear cracks subject to antiplane deformations," in Proceedings of the 2nd International Scientific and Technical Conference Architecture and Construction Contemporary Problems, vol. 2, pp. 295-303, 2010.

[31] N. Gerolymos and G. Gazetas, "Development of Winkler model for static and dynamic response of caisson foundations with soil and interface nonlinearities," Soil Dynamics and Earthquake Engineering, vol. 26, no. 5, pp. 363-376, 2006.

[32] D. S. Dugdale, "Yielding of steel sheets Containing slits," Journal of the Mechanics and Physics of Solids, vol. 8, no. 2, pp. 100-104, 1960.

[33] G. I. Barenblatt, "The mathematical theory of equilibrium cracks in brittle fracture," in Advances in Applied Mechanics, vol. 7, pp. 55-129, Academic Press, 1962.

[34] A. G. Pahlaviani, "Main characteristics of fracture failure of a piecewise homogeneous elastic plate with crack subject to antiplane deformations," in Proceedings of the 2nd International Scientific and Technical Conference Architecture and Construction Contemporary Problems, vol. 2, pp. 304-310, 2010.

[35] P. S. Theocaris and S. A. Paipetis, "State of stress around inhomogeneities by the method of caustics," Fibre Science and Technology, vol. 9, no. 1, pp. 19-39, 1976.

[36] P. S. Theocaris and S. A. Paipetis, "Constrained zones at singular points of inclusion contours," International Journal of Mechanical Sciences, vol. 18, no. 11-12, pp. 581-587, 1976. 


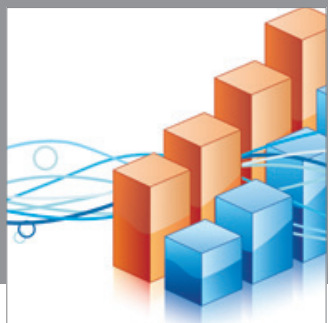

Advances in

Operations Research

mansans

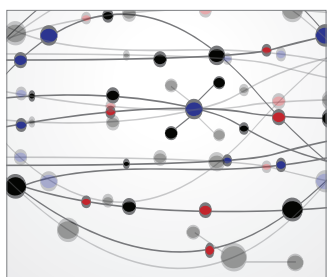

The Scientific World Journal
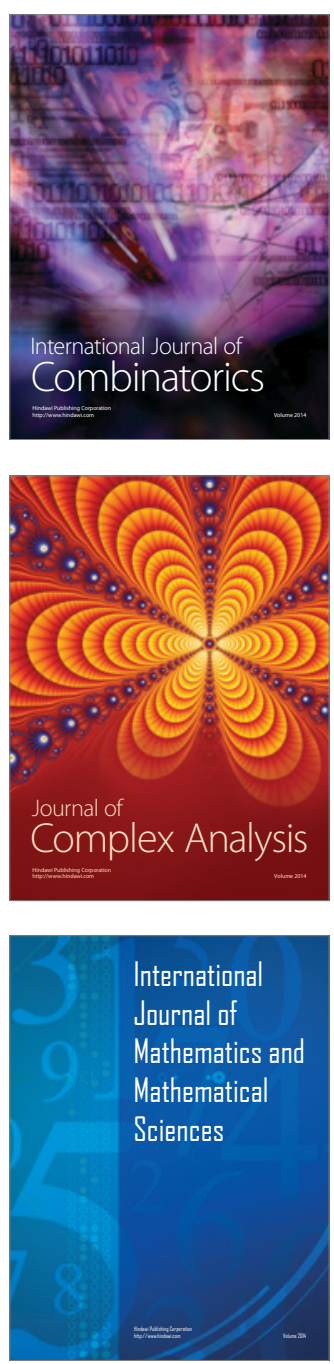
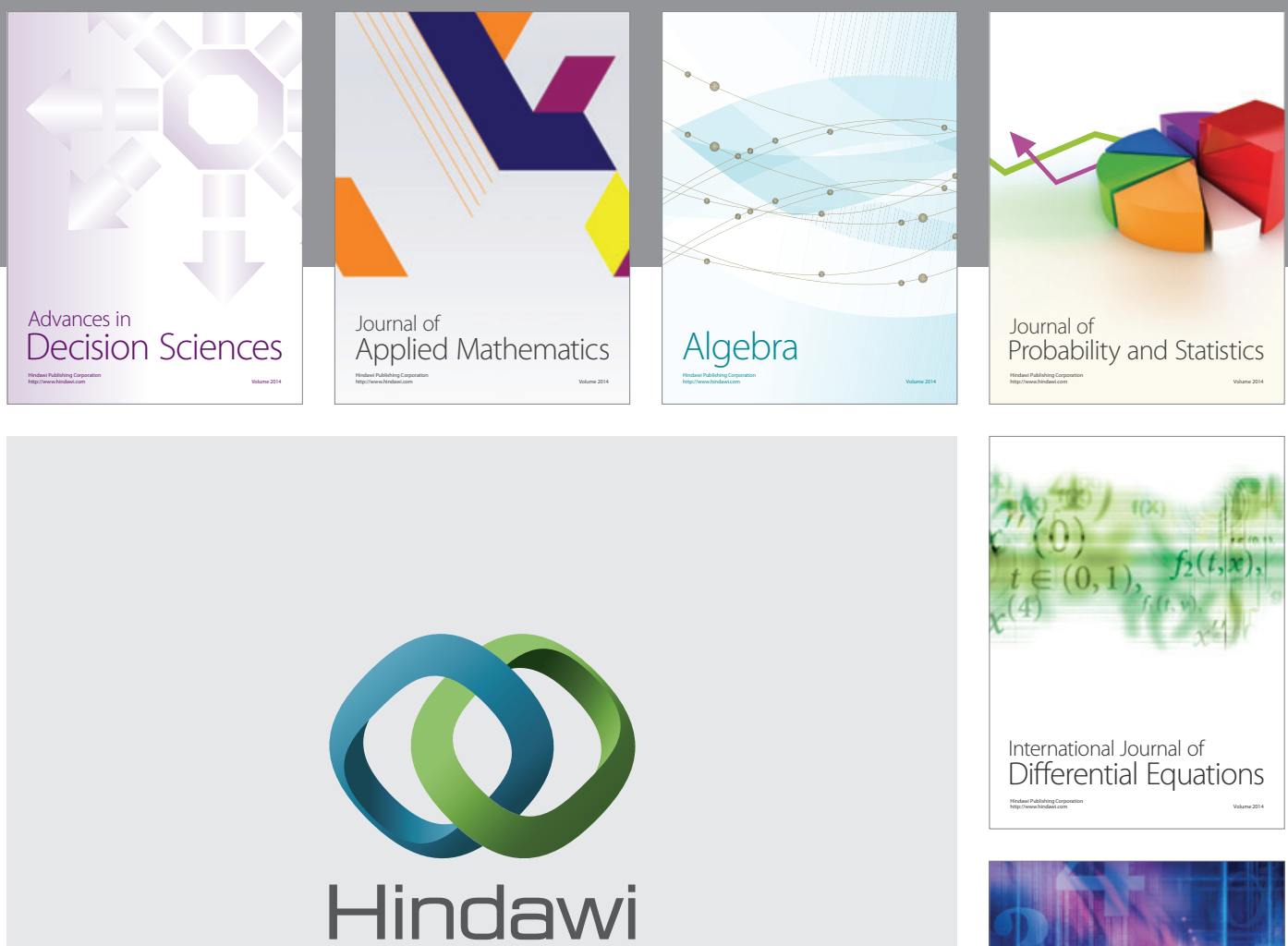

Submit your manuscripts at http://www.hindawi.com
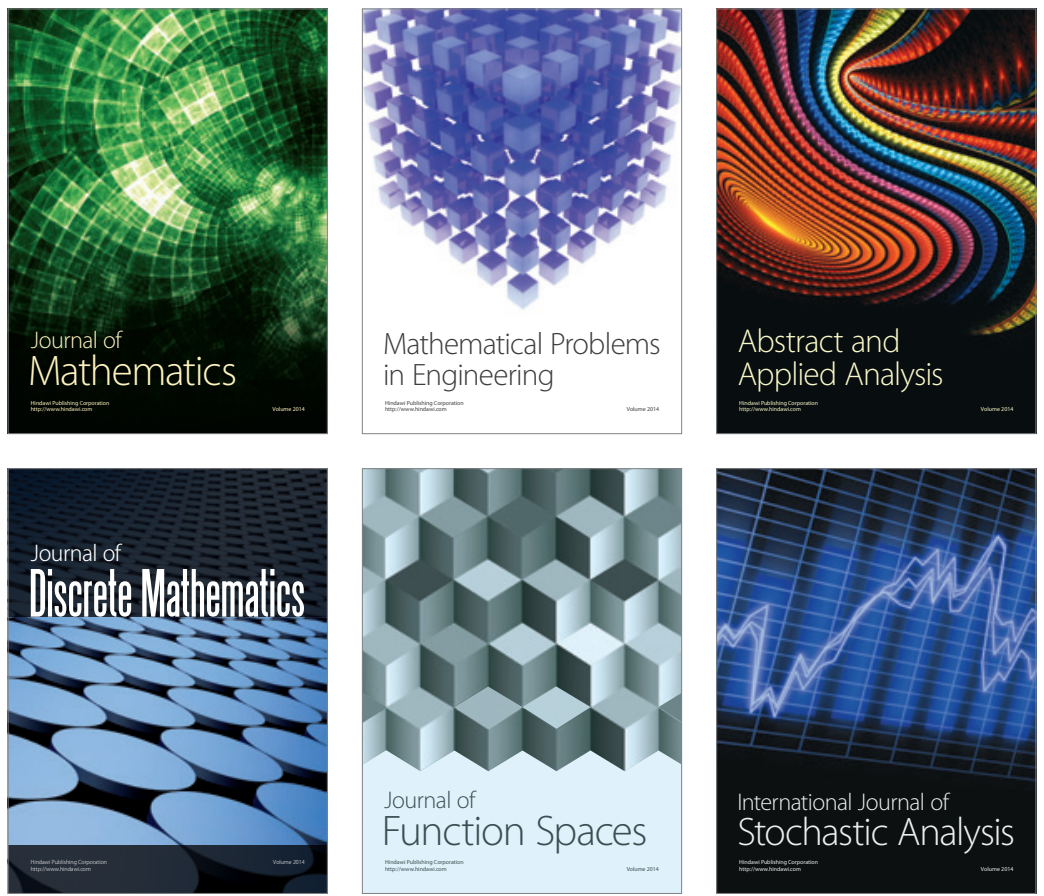

Journal of

Function Spaces

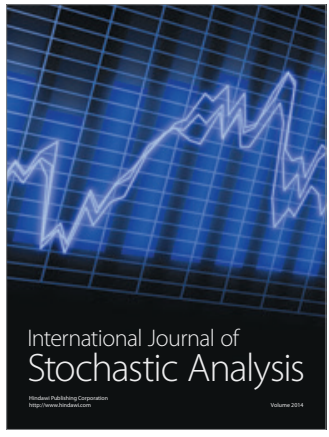

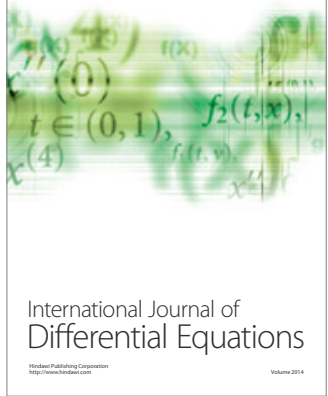
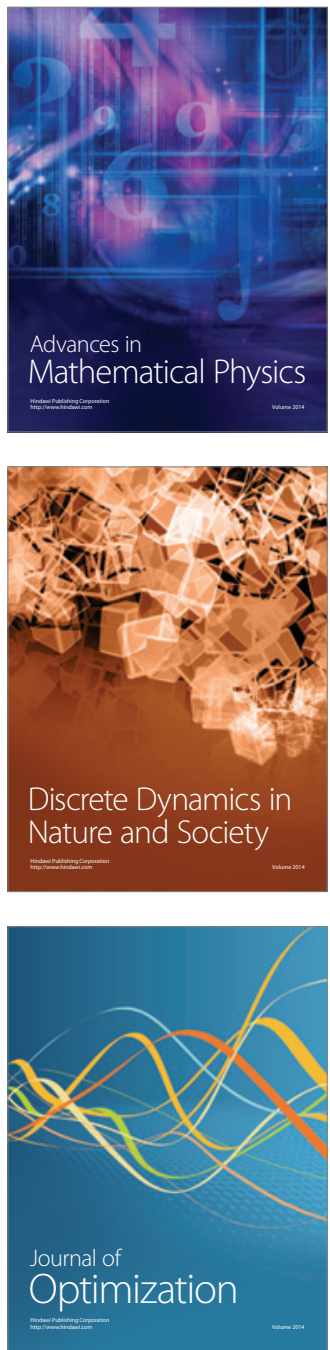